\title{
Metal enrichment of the neutral gas of blue compact dwarf galaxies: the compelling case of Pox 36
}

\author{
V. Lebouteiller ${ }^{1}$, D. Kunth ${ }^{2}$, T. X. Thuan ${ }^{3}$, and J. M. Désert ${ }^{2}$ \\ ${ }^{1}$ Center for Radiophysics and Space Research, Cornell University, Space Sciences Building, Ithaca, NY 14853-6801, USA \\ e-mail: vianney@isc.astro.cornell.edu \\ 2 Institut d'Astrophysique de Paris, 98bis Boulevard Arago, 75014 Paris, France \\ 3 Astronomy Department, University of Virginia, Charlottesville, VA 22903, USA
}

Received 4 October 2008 / Accepted 11 December 2008

\section{ABSTRACT}

\begin{abstract}
Context. Evidence has grown over the past few years that the neutral phase of blue compact dwarf (BCD) galaxies may be metaldeficient as compared to the ionized gas of their $\mathrm{H}$ II regions. These results have strong implications for our understanding of the chemical evolution of galaxies, and it is essential to strengthen the method, as well as to find possible explanations.

Aims. We present the analysis of the interstellar spectrum of Pox 36 with the Far Ultraviolet Spectroscopic Explorer (FUSE). Pox 36 was selected because of the relatively low foreground gas content that makes it possible to detect absorption-lines weak enough that unseen components should not be saturated.

Methods. Interstellar lines of H I, N I, O I, Si II, P II, Ar I, and Fe II are detected. Column densities are derived directly from the observed line profiles except for $\mathrm{H}$ I, whose lines are contaminated by stellar absorption, thus needing the stellar continuum to be removed. We used the TLUSTY models to remove the stellar continuum and isolate the interstellar component. The best fit indicates that the dominant stellar population is B0. The observed far-UV flux agrees with an equivalent number of $\sim 300 \mathrm{~B} 0$ stars. The fit of the interstellar $\mathrm{H}$ I line gives a column density of $10^{20.3 \pm 0.4} \mathrm{~cm}^{-2}$. Chemical abundances were then computed from the column densities using the dominant ionization stage in the neutral gas. Our abundances are compared to those measured from emission-line spectra in the optical, probing the ionized gas of the $\mathrm{H}$ II regions.

Results. Our results suggest that the neutral gas of Pox 36 is metal-deficient by a factor $\sim 7$ as compared to the ionized gas, and they agree with a metallicity of $\approx 1 / 35 Z_{\odot}$. Elemental depletion is not problematic because of the low dust content along the selected lines of sight. In contrast, the ionized gas shows a clear depletion pattern, with iron being strongly depleted.

Conclusions. The abundance discontinuity between the neutral and ionized phases implies that most of the metals released by consecutive star-formation episodes mixes with the $\mathrm{H}$ I gas. The volume extent of the enrichment is so large that the metallicity of the neutral gas increases only slightly. The star-forming regions could be enriched only by a small fraction $(\sim 1 \%)$, but it would greatly enhance its metallicity. Our results are compared to those of other BCDs. We confirm the overall underabundance of metals in their neutral gas, with perhaps only the lowest metallicity BCDs showing no discontinuity.
\end{abstract}

Key words. ISM: abundances - ISM: HII regions - line: profiles - galaxies: abundances - galaxies: dwarf galaxies: individual: Pox 36

\section{Introduction}

In the chemical downsizing scenario, in which massive galaxies are the first to form stars at a high rate (e.g., Cen \& Ostriker 1999), dwarf galaxies can remain chemically unevolved until later times. It is thus theoretically possible that some dwarf galaxies in the nearby Universe still contain pristine gas. The first observational constraint was provided by studies of chemical abundances in the ionized gas of blue compact dwarf galaxies (BCDs). These studies yield a fundamental result: a metallicity floor appears to exist, with no BCD having an ionized gas oxygen abundance lower than $12+\log (\mathrm{O} / \mathrm{H}) \sim 7.0$, or $Z_{\odot} / 50^{1}$. For more than three decades, I $\mathrm{Zw} 18$ discovered by Zwicky (1966) and first studied by Sargent \& Searle (1970) held the record as the most metal-deficient galaxy known, with an oxygen abundance $12+\log (\mathrm{O} / \mathrm{H})=7.17 \pm 0.01$ in its NW component and $7.22 \pm 0.02$ in its SE component (Thuan \& Izotov 2005). Only very recently has IZw 18 been displaced by two

${ }^{1}$ We use hereafter the solar oxygen abundance $12+\log (\mathrm{O} / \mathrm{H})=8.66$ of Asplund et al. (2005)
BCDs that are more metal-deficient, SBS 0335-052W with $12+$ $\log (\mathrm{O} / \mathrm{H})=7.12 \pm 0.03$ (Izotov et al. 2005) and DDO 68 with $12+\log (\mathrm{O} / \mathrm{H})=7.14 \pm 0.03$ (Izotov \& Thuan 2007). The difficulty of finding more metal-poor objects was originally explained by the local mixing of the amount of metals produced and released in a single starburst episode (Kunth \& Sargent 1986). This hypothesis has been since challenged by observations of uniform abundances in disconnected star-forming regions within a single dwarf galaxy (e.g., Skillman \& Kennicut 1993; Kobulnicky \& Skillman 1997; Noeske et al. 2000).

Tenorio-Tagle (1996) proposed an attractive scenario in which supernovae ( $\mathrm{SNe}$ ) products are driven to high galactic latitudes in a hot phase $\left(\sim 10^{6} \mathrm{~K}\right)$ before they cool down, condense as molecular droplets, and rain back on the disk. The complete cycle (before mixing) would last several $\sim 100 \mathrm{Myr}$. This scenario naturally explains the uniform abundances found in dwarf galaxies, while ruling out local enrichment within the star-forming regions. It must be noted that models of Rieschick \& Hensler $(2001,2003)$, based on Tenorio-Tagle's scenario, predict that only $\sim 75 \%$ of the SNe products go through the outer 
galactic cycle as hot gas, while the remaining $25 \%$ evolve in an inner local cycle and can enrich the star-forming regions in only $10 \mathrm{Myr}$. A few percent could eventually leave the gravitational field of the galaxy. On the other hand, Chandra X-ray observations of SBS 0335-052W, SBS 0335-052E, and IZw 18 do not reveal any sign of a hot gas breaking out from the stellar body implying that the galactic fountain scenario may not be valid for BCDs (Thuan et al. 2004). In fact, Recchi et al. (2001, 2004) modeled star-formation in gas-rich dwarf galaxies and found that the majority of newly produced metals resides in a cold phase $\left(\lesssim 2 \times 10^{4} \mathrm{~K}\right)$ after only a few tens of Myr, supporting the common instantaneous mixing hypothesis.

To understand the metal enrichment of galaxies, it is necessary to study all the gaseous phases involved in the gas mixing cycle. X-ray observations of starburst galaxies led to the conclusion that the hot gas is probably enriched by SNe products (e.g., Martin et al. 2002). The following step in the mixing cycle is poorly constrained observationally. Do metals mix with the cold halo and thus enriching the $\mathrm{H}$ I region? Do they eventually condense as molecular droplets and settle back onto the stellar body? The situation might be different in BCDs if galactic fountains do not form. However, it seems that winds from massive stars and SNe are still able to carve the ISM to form superbubbles in which metals are injected at high temperatures (Thuan et al. 2004). Mixing could then occur at the interfaces between the hot and cold gas. Thus the study of the metal content in the neutral gas of dwarf galaxies provides us with essential constraints for their chemical evolution models.

The neutral phase usually consists of at least two distinct components (Field et al. 1969), with a warm medium (WNM; $T_{\mathrm{w}} \sim 10^{4} \mathrm{~K}, n_{\mathrm{w}} \sim$ few tenths $\mathrm{cm}^{-3}$ ) showing broad hyperfine $21 \mathrm{~cm}$ line emission, and a cold medium (CNM, $T_{\mathrm{c}} \sim 100 \mathrm{~K}$, $n_{\mathrm{c}} \sim$ few tens $\mathrm{cm}^{-3}$ ) showing narrow $21 \mathrm{~cm}$ emission and absorption. It must be noted that the distinction between CNM and WNM becomes indistinct in galaxies with low-pressure ISM, such as dwarfs (Lo et al. 1993). Neutral metals can be observed through resonant lines in the far-ultraviolet (FUV). BCDs are ideal targets because they display large amounts of $\mathrm{H}$ I gas (Thuan \& Martin 1981), and because the massive stars provide a strong FUV continuum. Kunth et al. (1994) used the GHRS instrument on board the Hubble Space Telescope to observe interstellar absorption lines along the lines of sight toward the many massive stars in IZw18. The authors detected the O I line at $1302.2 \AA$ and, together with the neutral hydrogen content measured from the $21 \mathrm{~cm}$ line, estimated the metallicity of the neutral envelope to be at least 10 times lower than that of the ionized gas in the H II regions. Later, Thuan \& Izotov (1997) obtained a GHRS spectrum for SBS 0335-052E and found the metallicity of the neutral gas to be similar to that of the ionized gas, about $2 \%$ solar. These results remained however inconclusive because of possible saturation effects of the $\mathrm{O}$ I absorption line (see Pettini \& Lipman 1995).

A new step forward was achieved with the launch of the Far Ultraviolet Spectroscopic Explorer (FUSE; Moos et al. 2000), which allows the observation of absorption-lines of $\mathrm{H}$ I together with many metallic species such as N I, O I, Si II, $\mathrm{P}$ II, Ar I, and Fe II. The spectral resolution of FUSE $(R \sim$ 20000 ) and its sensitivity made it possible to detect numerous absorption-lines, some of them apparently not saturated. The neutral gas chemical composition was derived in several $\mathrm{BCD}$, spanning a wide range in ionized gas metallicity, from $1 / 50$ to $1 / 3$ solar, with IZw 18 (Aloisi et al. 2003; Lecavelier des Etangs et al. 2004), SBS 0335-052 (Thuan et al. 2005), IZw 36 (Lebouteiller et al. 2004), Mark 59 (Thuan et al. 2002), NGC 625
(Cannon et al. 2005), and NGC 1705 (Heckman et al. 2001). These investigations showed that the neutral gas of BCDs is not pristine, but that it has already been enriched with metals up to an amount of $\gtrsim 1 / 50 Z_{\odot}$. The second most important result is that the metallicity of the neutral gas is generally lower than that of the ionized gas - except for the two lowest-metallicity BCDs, IZw 18 and SBS 0335-052E - implying that the neutral phase has been probably less processed.

The interpretation of these remarkable results has been limited so far by the lack of knowledge about the origin of the neutral gas in BCDs whose absorption is detected in the FUV. Direct comparison with the $\mathrm{H}$ I gas seen via the $21 \mathrm{~cm}$ emission is hampered by a bias in selecting out relatively dust-free FUV lines of sight toward the massive stars. Even more importantly, FUV observations only probe gas in front of stellar clusters while radio observations in emission probe the whole system. Many uncertainties also remain concerning the method of deriving column densities from absorption-lines in complex systems. Most FUSE studies of BCDs assumed a single homogeneous line of sight with the exception of Mark 59 (Thuan et al. 2002; see also Lebouteiller et al. 2006 for the giant H II region NGC 604). A more realistic approach would be to consider multiple lines of sight toward massive stars, each line of sight intersecting clouds with possibly different physical conditions (such as turbulent velocity $b$, radial velocity $v$ ) and chemical properties. If individual absorption components are unresolved, it is almost impossible to tell whether they are saturated, in which case the column density inferred from the global absorption line can be severely underestimated (see e.g., Lebouteiller et al. 2006). Following the suggestion of Kunth \& Sargent (1986), Bowen et al. (2005) got around the problem by deriving the abundances in the neutral gas of the dwarf spiral galaxy SBS 1543+593 along a quasar line of sight. The authors found that the abundances agree with those in the ionized gas of the brightest $\mathrm{H}$ II region in the arms, contrasting with the FUSE results in BCDs. As far as chemical models are concerned, Recchi et al. (2004) examined the case of IZw 18 for which they found that abundances in the $\mathrm{H}$ I medium (defined by a temperature lower than $7000 \mathrm{~K}$ ) are similar to those in the H II gas. Hence, although some physics is still missing in the models (radiative transfer, clumpy ISM, dust phase), it seems that FUSE results still cannot be reproduced.

In this paper, we look anew at the problem of how the chemical compositions of the ionized and the neutral gas in BCDs compare by selecting an object with an extremely low H I column density. This object has some absorption lines that are weak enough to be safely considered as unsaturated. The object we analyze is Pox 36 (also known as ESO 572-G034, I SZ 63, HIPASSJ1158-19b, or IRAS 11564-1844), an emissionline galaxy first studied by Rodgers et al. (1978) and then rediscovered by Kunth et al. (1981). It is characterized by strong optical emission-lines superimposed on a very blue continuum. Its spectral characteristics and morphological properties fit the definition of a BCD galaxy (see e.g., Thuan \& Martin 1981; Kunth \& Östlin 2000). Its known observational properties are listed in Table 1. In particular, chemical abundances in the ionized gas of the $\mathrm{H}$ II regions within Pox 36 have been derived by several independent studies (e.g., Kunth \& Sargent 1983; Izotov \& Thuan 2004). The ionized gas has an oxygen abundance $12+\log (\mathrm{O} / \mathrm{H}) \approx 8.05$ which corresponds to $Z_{\odot} / 5$. With a $\mathrm{H}$ I velocity of $1114 \mathrm{~km} \mathrm{~s}^{-1}$ (Meyer et al. 2004), a Hubble constant of $73 \mathrm{~km} \mathrm{~s}^{-1} \mathrm{Mpc}^{-1}$, its cosmology-corrected distance is $20 \mathrm{Mpc}$.

The FUSE observations are presented in Sect. 2 and an overview of the FUSE spectrum is given in Sect. 3. We infer the 


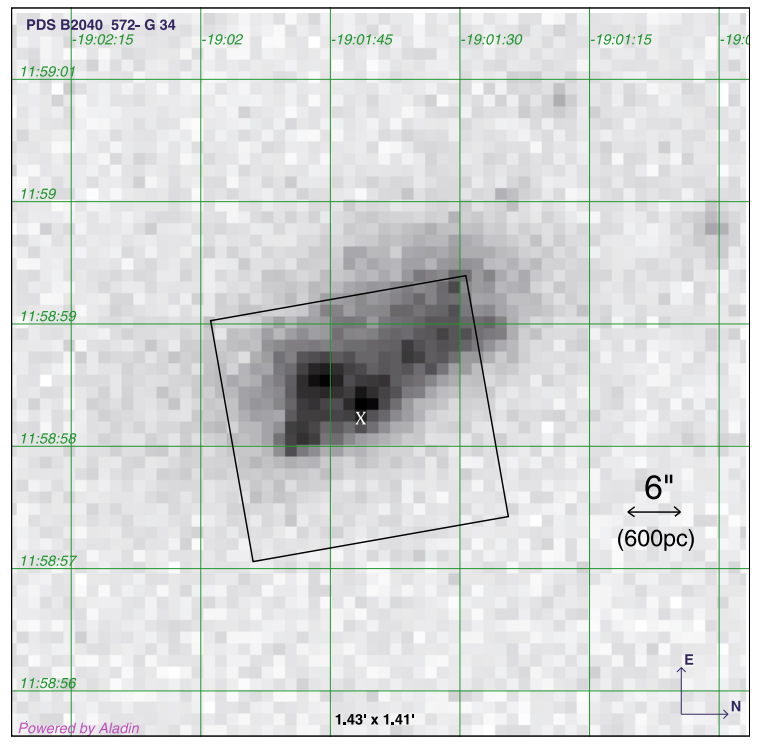

Fig. 1. Blue band image of Pox 36 (Astro-Wise, ESO-LV Catalog). The separation between the 2 bright knots is given by the segment. The inclined square indicates the $30^{\prime \prime} \times 30^{\prime \prime}$ LWRS aperture.

column densities of $\mathrm{H}$ I and of the metals from the line profile in Sect. 4. Chemical abundances are derived and discussed in Sect. 5. Finally, we study the properties of the neutral gas in the current FUSE BCD sample to discuss general trends in Sect. 7.

\section{Observations}

Pox 36 was observed by the FUSE telescope on May 112001 (observation B0220201). All eight observing channels were used with the LWRS aperture $\left(30^{\prime \prime} \times 30^{\prime \prime}\right)$. At the distance of Pox 36 (see Table 1 ), $1^{\prime \prime}$ corresponds to $\approx 100 \mathrm{pc}$. The total exposure time is 25470 s. Data were taken in "Time Tag" observing mode. The brightest blue regions are included in the aperture as well as the fainter extended emission seen toward the north-east (Fig. 1). In the blue band, the combined emission from the bright knots is on the same order as the emission from the rest of the body. We expect shorter wavelength light such as the FUV emission to be dominated by the knots where most of the massive stars lie.

Data were processed by the CalFUSE ${ }^{2}$ pipeline (version 3.2.1). We aligned the individual exposures on the wavelength grid and co-added them to obtain one spectrum per observing channel. The final spectrum is presented in Fig. 2, in which observations from different channels have been co-added for display purposes. The signal-to-noise ratio per resolution element $\left(20 \mathrm{~km} \mathrm{~s}^{-1}\right)$ is $\approx 12$.

The intrinsic spectroscopic resolving power of FUSE is close to $R=20000$ corresponding to a line spread function (LSF) width of $\sim 15 \mathrm{~km} \mathrm{~s}^{-1}$ (e.g., Sahnow et al. 2000; Hébrard et al. 2002; Kruk et al. 2002). The effective resolution depends on the quality of the individual exposure alignment. In particular, the alignment is difficult to assess for spectra in the $\mathrm{SiC}$ channels because of the smaller effective area as compared to the LiF channels, resulting in a lower signal-to-noise ratio. Misalignments reduce significantly the spectral resolution of the final spectrum so that we estimate the LSF width to be as large as $\sim 25 \mathrm{~km} \mathrm{~s}^{-1}$.

The spatial extent of the source within the LWRS aperture can also result in a smearing of the spectrum (see e.g.,

2 The CalFUSE calibration pipeline is available at http://fuse. pha.jhu.edu/analysis/calfuse.html
Lebouteiller et al. 2004, 2006). Unfortunately, Pox 36 has not been imaged in the UV. The optical blue image of Pox 36 shows two bright knots separated by $\approx 6^{\prime \prime}$ (Fig. 1), corresponding to a projected distance of $\approx 4^{\prime \prime}$ in the dispersion direction. Given the LWRS aperture size of $30^{\prime \prime}=120 \mathrm{~km} \mathrm{~s}^{-1}$, the separation between the 2 knots should result in a wavelength smearing of $\sim 16 \mathrm{~km} \mathrm{~s}^{-1}$ at most.

Since the line broadening also depends on several observational parameters (such as the pointing jitter), it is difficult to derive a priori a precise estimate. We empirically determined the LSF width by using the separation between closed or blended absorption lines. The most likely value is $\sim 30 \mathrm{~km} \mathrm{~s}^{-1}$ for the LiF channels and $\sim 37.5 \mathrm{~km} \mathrm{~s}^{-1}$ for the SiC channels. This is roughly consistent with the quadratic sum of the intrinsic PSF and the smearing due to the spatial extent. This is the LSF width we use for line fitting (Sect. 4.1). In practice, our results depend little on the LSF width determination (see Sect. 4.1.1).

\section{Overview of the FUSE spectrum}

\subsection{Absorption systems}

The spectral continuum is provided by the UV-bright massive stars in the galaxy. Absorption lines from species along the line of sight are superimposed on the continuum. The line of sight intersects the ISM from the Milky Way and from Pox 36 itself. As noted by Wakker et al. (2003), the line of sight passes at only $18^{\prime}(\approx 100 \mathrm{kpc}$ projected) from NGC 4027 , but there is no absorption visible at the velocity of the latter $\left(\approx 1460 \mathrm{~km} \mathrm{~s}^{-1}\right)$. The redshift of Pox 36 makes it possible to separate easily the local absorption system from the one in the BCD. We identify absorption lines from the Milky Way at nearly zero radial velocity. We barely detect Galactic $\mathrm{H}_{2}$, which is mostly due to the high Galactic latitude of Pox 36 (Table 1).

The absorption system corresponding to the neutral ISM in Pox 36 is detected at a velocity of $v_{\mathrm{n}} \sim 1052 \mathrm{~km} \mathrm{~s}^{-1}(1058 \pm$ $10 \mathrm{~km} \mathrm{~s}^{-1}$ from the metal lines, $1047 \pm 10 \mathrm{~km} \mathrm{~s}^{-1}$ from the $\mathrm{H}$ I lines). This corresponds to a wavelength shift of $\sim 3.5-4.0 \AA$ from the rest-frame. Only the strongest atomic lines are detected. $\mathrm{No} \mathrm{H}_{2}$ line at the redshift of Pox 36 is seen. The velocity $v_{\mathrm{n}}$ is significantly smaller than the one derived from the $21 \mathrm{~cm} \mathrm{H}$ I line equal to $1113.9 \pm 6.5 \mathrm{~km} \mathrm{~s}^{-1}$ (Meyer et al. 2004; Zwaan et al. 2004). However, it must be stressed that the regions probed in the UV and the radio are different in terms of extent (the radio beam size is $15.5^{\prime}$ as compared to the $30^{\prime \prime} \times 30^{\prime \prime}$ aperture of the LWRS) and of depth (because of dust extinction). The aperture (and depth) effect explanation is supported by the fact that $v_{\mathrm{n}}$ is in agreement with the value of $1065 \pm 20 \mathrm{~km} \mathrm{~s}^{-1}$ derived by Izotov \& Thuan (2004) from the optical emission lines of the ionized gas (Table 1). Taking at face the velocity of the neutral and ionized gas tracers, we conclude that there is no significant evidence that the neutral gas is outflowing from the star-forming regions.

We find that the turbulent velocity of the neutral gas in Pox 36 is $b_{\mathrm{n}}=33 \pm 10 \mathrm{~km} \mathrm{~s}^{-1}$. Its value is mostly constrained by lines near saturation such as the O I $988.77 \AA$ line (Table 2). The quoted uncertainty on the $b$-value does not include possible systematic errors on the LSF width. The corresponding FWHM is $2 b_{\mathrm{n}} \sqrt{\ln 2} \approx 55 \pm 17 \mathrm{~km} \mathrm{~s}^{-1}$, consistent with the $F W H M$ measured in radio $\left(67.4 \mathrm{~km} \mathrm{~s}^{-1}\right.$, Table 1$)$. However as said before, the region probed by the large radio beam $\left(15.5^{\prime}\right)$ is not directly comparable to the gas probed in the FUV (corresponding to an extent of less than $\sim 10^{\prime \prime}$, Fig. 1 ). It must be also stressed that our determination of $b_{\mathrm{n}}$ may not be physical if the absorption 


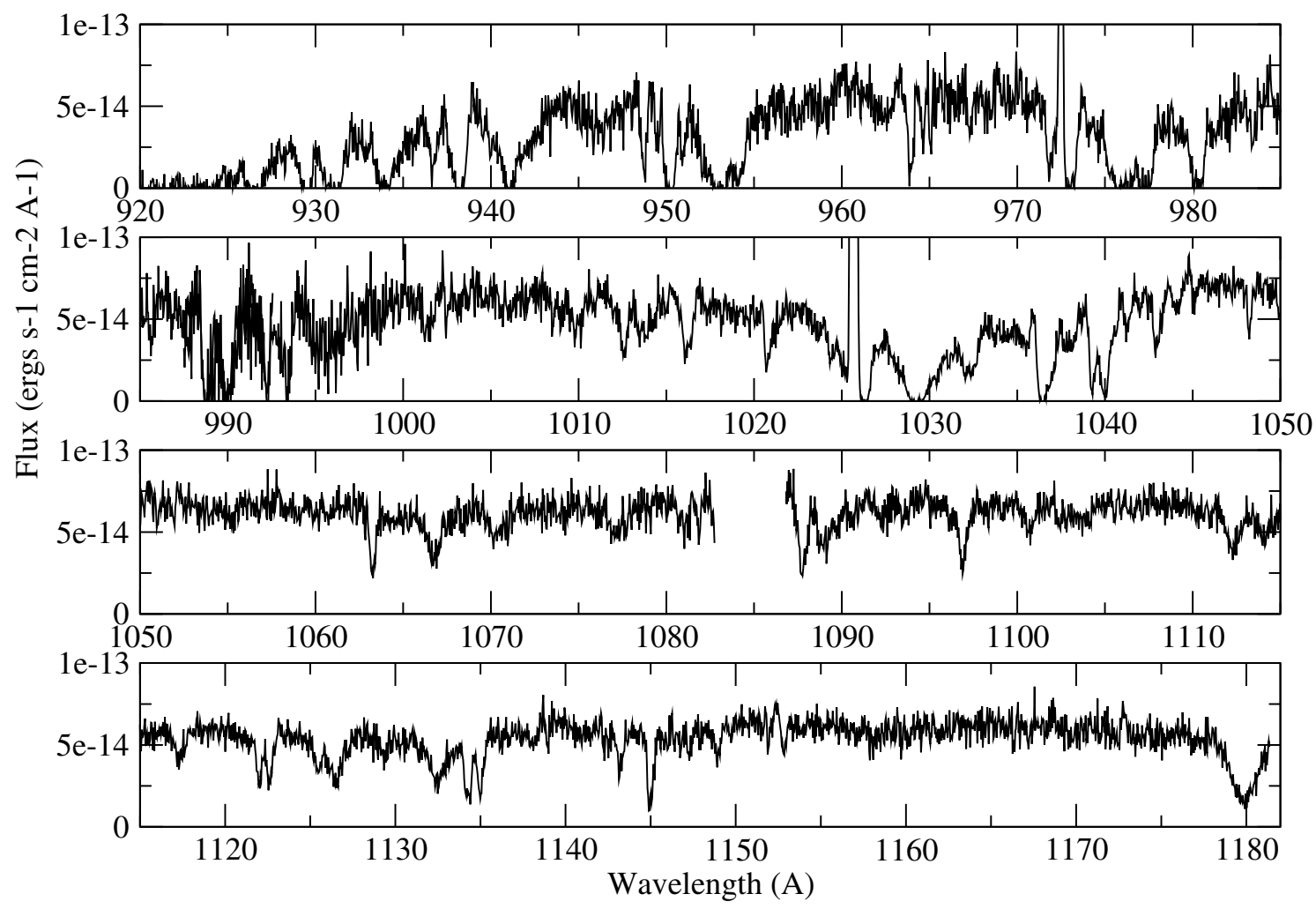

Fig. 2. Complete FUSE spectrum of Pox 36. The flux is plotted against the observed wavelength (3.5-4 $\AA$ shift from the rest wavelength, Sect. 3.1). Data from overlapping channels were co-added for display purposes. The strong emission-lines at $\sim 972 \AA$ and $\sim 1026 \AA$ correspond to terrestrial H I airglows.

lines arise from a collection of interstellar clouds (see Sects. 3.2 and 4.1.2).

\subsection{Neutral ISM components}

The lines of sight observed by FUSE are optically thick to the Lyman continuum of the stellar clusters but the UV radiation is still able to reach us. This implies that the amount of foreground dust is either very small and/or it is clumpy, as expected for metal-poor objects (e.g., Lisenfeld \& Ferrara 1998; Hirashita et al. 2002). Although FUSE observations are sensitive to both the CNM and the WNM as defined in McKee \& Ostriker (1977) and Wolfire et al. (1995), the situation might be complex in BCDs since the relatively low ISM pressure could lead to a single H I phase, except in regions affected by stellar winds and supernovæ shocks. Unfortunately, the spectral resolution of FUSE (Sect. 2) is too small to isolate warm and cold neutral phases.

In principle, the presence of molecular absorption could allow us to constrain the physical conditions along the observed lines of sight. No diffuse $\mathrm{H}_{2}$ has been detected so far along lines of sight toward massive stars in BCDs (e.g., Vidal-Madjar et al. 2000). The reasons are intrinsic to the $\mathrm{H}$ I phase since $\mathrm{H}_{2}$ has been detected in emission in the warm ISM of these objects (e.g., Vanzi et al. 2005; Thuan et al. 2005). Warm $\mathrm{H}_{2}$ has been since observed in several other BCDs by the Infrared Spectrograph onboard Spitzer (Lebouteiller et al. in preparation). The absence of $\mathrm{H}_{2}$ in the diffuse ISM of BCDs is due to the combined effects of a low $\mathrm{H}$ I density in the neutral gas envelope, a large UV flux that destroys $\mathrm{H}_{2}$ molecules and a low metallicity that makes grains on which to form $\mathrm{H}_{2}$ molecules scarce (Vidal-Madjar et al. 2000). Diffuse $\mathrm{H}_{2}$ is detected in relatively more metal-rich star-forming objects, but the molecular fraction is very low, on the order of $10^{-4}$ (Hoopes et al. 2004). A small velocity offset $\left(\sim 10 \mathrm{~km} \mathrm{~s}^{-1}\right)$ between $\mathrm{H}_{2}$ lines and atomic lines has been found in NGC 625 (Cannon et al. 2005) and toward the giant H II region NGC 604 (Lebouteiller et al. 2006). Although the offset is small, this might indicate that atomic absorption-lines preferentially arise in regions where $\mathrm{H}_{2}$ is not able to form. Hence, it seems reasonable to assume that the neutral phase probed by FUV absorption-lines is rather warm and diffuse since dense clouds should show $\mathrm{H}_{2}$ absorption. In the following, we thus assume that the interstellar absorption-lines arise from a single phase dominated by turbulent motions. Since the filling factor is unknown, this phase could be a uniform H I envelope or a collection of $\mathrm{H}$ I clouds.

\subsection{Stellar contamination}

The FUV spectral continuum of most of the BCDs and giant $\mathrm{H}$ II regions studied so far with FUSE is dominated by $\mathrm{O}$ stars. This is indicative of a starburst age younger than $\sim 10 \mathrm{Myr}$ (Robert et al. 2003). O stars have relatively "clean" spectra, the most prominent stellar absorption lines being due to He II, O IIIII, and N II-III in the optical, and to O VI, N V, P V, S IV, C IV and Si IV in the FUV. Because of the high temperature, hydrogen is mostly ionized, and only weak H I photospheric lines can be observed which contribute to the - already saturated - core of the interstellar H I absorption line. Hence FUV spectra toward young starbursts allow a precise determination of the interstellar H I content. The only exception concerns extremely young starburst episodes showing a prominent O VI P-Cygni profile, which complicates a reliable profile fitting of the wings of Ly $\beta$ (Lebouteiller et al. 2006). 
Table 1. Observational properties of Pox 36.

\begin{tabular}{|c|c|c|}
\hline Property & Value & Comment \\
\hline$\alpha(\mathrm{J} 2000)$ & $11 \mathrm{~h} 58 \mathrm{~m} 57.3 \mathrm{~s}$ & HIPASS (Meyer et al. 2004) \\
\hline$\delta(\mathrm{J} 2000)$ & $-19^{\circ} 01^{\prime} 41^{\prime \prime}$ & HIPASS (Meyer et al. 2004) \\
\hline Galactic coordinates & $l=286.114, b=42.123$ & HIPASS (Meyer et al. 2004) \\
\hline Foreground Galactic extinction (mag) & $E(B-V)=0.038$ & Schlegel et al. (1998) \\
\hline Magnitude (mag) & $\begin{array}{l}B_{J}=14.27, I=14.38 \\
K_{\mathrm{s}}=12.63\end{array}$ & $\begin{array}{l}\text { HIPASS (Doyle et al. 2005) } \\
\text { Vanzi \& Sauvage (2006) }\end{array}$ \\
\hline$L(\mathrm{H} \beta)\left(\mathrm{erg} \mathrm{s}^{-1}\right)$ & $1.81 \times 10^{39}$ & From $F(\mathrm{H} \beta)$ (Izotov \& Thuan 2004) and $D=20 \mathrm{Mpc}$ \\
\hline$F W H M(\mathrm{H} \mathrm{I})\left(\mathrm{km} \mathrm{s}^{-1}\right)$ & 67.4 & HIPASS (Meyer et al. 2004) \\
\hline Peak H I flux (Jy) & 0.1197 & HIPASS (Meyer et al. 2004) \\
\hline Integrated $\mathrm{H}$ I flux $\left(\mathrm{Jy} \mathrm{km} \mathrm{s}^{-1}\right)$ & 8.6 & HIPASS (Meyer et al. 2004) \\
\hline$M(\mathrm{H} \mathrm{I})\left(M_{\odot}\right)$ & $8.1 \times 10^{8}$ & Derived from the $\mathrm{H}$ I flux with the distance $=20 \mathrm{Mpc}$ \\
\hline Radial velocity $\left(\mathrm{km} \mathrm{s}^{-1}\right)$ & $\begin{array}{l}1114 \pm 2 \\
1113.9 \pm 6.5 \\
1065 \pm 20\end{array}$ & $\begin{array}{l}21 \mathrm{~cm} \text { line, Theureau et al. (1998) } \\
\text { HIPASS (Meyer et al. 2004; Zwaan et al. 2004) } \\
\text { Optical lines (Izotov \& Thuan 2004) }\end{array}$ \\
\hline Distance (Mpc) & $\begin{array}{l}\approx 12 \\
\approx 16 \\
\approx 20\end{array}$ & $\begin{array}{l}\text { Vigroux et al. (1987) with } H_{0}=73 \mathrm{~km} \mathrm{~s}^{-1} \\
\text { Kunth \& Joubert (1985) } \\
\text { Using } v=1114 \mathrm{~km} \mathrm{~s}^{-1}, H_{0}=73 \mathrm{~km} \mathrm{~s}^{-1}, \Omega_{\mathrm{m}}=0.27\end{array}$ \\
\hline $\log \mathrm{O} / \mathrm{H}$ & $\begin{array}{l}-3.95 \pm 0.02 \\
-3.86 \pm 0.03 \\
-3.81 \pm 0.04 \\
-3.82 \pm 0.04\end{array}$ & $\begin{array}{l}\text { Izotov \& Thuan (2004) } \\
\text { Kobulnicky \& Skillman (1996) } \\
\text { Campbell (1988, 1992) } \\
\text { Kunth \& Sargent (1983) }\end{array}$ \\
\hline $\log \mathrm{N} / \mathrm{H}$ & $\begin{array}{l}-5.53 \pm 0.03 \\
-5.39 \pm 0.10 \\
-5.38 \pm 0.05 \\
-5.37 \pm 0.05\end{array}$ & $\begin{array}{l}\text { Izotov \& Thuan (2004) } \\
\text { Kobulnicky \& Skillman (1996) } \\
\text { Campbell (1992) } \\
\text { Kunth \& Sargent (1983) }\end{array}$ \\
\hline $\begin{array}{l}\log \mathrm{Ar} / \mathrm{H} \\
\log \mathrm{Fe} / \mathrm{H}\end{array}$ & $\begin{array}{l}-6.24 \pm 0.03 \\
-6.17 \pm 0.10\end{array}$ & $\begin{array}{l}\text { Izotov \& Thuan (2004) } \\
\text { Izotov \& Thuan (2004) }\end{array}$ \\
\hline
\end{tabular}

Table 2. Wavelengths and oscillator strengths of the lines analyzed.

\begin{tabular}{llllll}
\hline \hline Species & $\lambda_{\text {rest }}(\AA)$ & $\lambda_{\text {obs }}(\AA)$ & $\log (g f)^{a}$ & $N_{\text {sat }}\left(\mathrm{cm}^{-2}\right)^{b}$ & Comment \\
\hline H I & 1025.722 & 1029.389 & 0.0527 & $\ldots$ & Lyman $\beta$ \\
H I & 937.803 & 941.080 & 0.0026 & $\ldots$ & Lyman $\epsilon$ \\
\hline N I $_{\text {N }}$ & 953.415 & 956.797 & 0.0132 & $5 \times 10^{14}$ & \\
N I & 953.655 & 957.031 & 0.0250 & $3 \times 10^{14}$ & \\
N I & 953.970 & 957.343 & 0.0350 & $2 \times 10^{14}$ & \\
N I & 963.990 & 967.040 & 0.0148 & $4 \times 10^{14}$ & Blended with P II $\lambda 963.8$ \\
N I & 964.626 & 968.029 & 0.0094 & $7 \times 10^{14}$ & Barely detected \\
N I & 965.041 & 968.458 & 0.0040 & $2 \times 10^{15}$ & Not detected \\
N I & 1134.165 & 1138.069 & 0.0134 & $4 \times 10^{14}$ & Barely detected \\
N I & 1134.415 & 1138.303 & 0.0268 & $2 \times 10^{14}$ & \\
\hline O I & 1134.980 & 1138.888 & 0.0402 & $8 \times 10^{13}$ & \\
O I & 988.773 & 992.209 & 0.0465 & $1 \times 10^{14}$ & Saturated \\
\hline Si II & 1039.230 & 1042.961 & 0.0092 & $5 \times 10^{14}$ & \\
\hline P II & 1020.699 & 1024.358 & 0.0164 & $2 \times 10^{14}$ & \\
P II & 963.800 & 967.249 & 1.4600 & $2 \times 10^{12}$ & Blended with N I $\lambda 963.99$ \\
\hline Ar I & 1152.818 & 1156.906 & 0.2450 & $2 \times 10^{13}$ & Not detected \\
\hline Fe II & 1048.220 & 1051.957 & 0.2630 & $9 \times 10^{12}$ & \\
\hline
\end{tabular}

${ }^{a}$ Atomic data are from Morton et al. (2003), except for Fe II $\lambda 1144.938$ for which we take Howk et al. (2000).

${ }^{b} N_{\text {sat }}$ is the maximum column density before saturation of a central component with $b=2 \mathrm{~km} \mathrm{~s}^{-1}$ (Sect. 4.1.2).

Older starbursts ( $\gtrsim 10 \mathrm{Myr})$ are dominated by B stars. Such stars have particularly strong He I lines in the optical, and overall a more complex FUV spectrum because the ionization degree is lower than in O stars. For this reason, photospheric H I lines becomes prominent (Valls-Gabaud 1993). As an illustration, the H I lines from the Lyman series in the BCD Mark 59 show "V-shaped" wings typical from B stars photospheres (Thuan et al. 2002). Pox 36 shows a similar pattern, but while
Thuan et al. (2002) got around the problem by considering an artificial continuum to fit the profile of the $\mathrm{H}$ I lines in Mark 59, we decided to model the stellar absorption in Pox 36 (Sect. 4.2).

\section{Column densities}

In order to infer chemical abundances, we derived column densities based on the line profiles. We adjusted the profiles of 
the metallic lines using the observed spectrum (Sect. 4.1). The $\mathrm{H}$ I column density was calculated from the Ly $\beta$ line in the spectrum corrected for stellar absorption (Sect. 4.2).

\subsection{Metals}

\subsubsection{Single component analysis}

For the profile fitting, we first made the usual assumption of a single line of sight intersecting a medium with uniform properties (namely with constant radial and turbulent velocities, and column densities). The actual situation can be more complex, with multiple lines of sight toward the many massive stars and $\mathrm{H}$ II regions within the galaxy (Sect. 4.1.2).

The method to derive column densities from FUSE line profiles is discussed in Hébrard et al. (2002). Profiles are adjusted using the Owens routine (Lemoine et al. 2002) through a minimization of the $\chi^{2}$ between the model and the observations. All the lines could be fitted with Voigt profiles. We considered that all the neutral species within Pox 36 share the same turbulent and radial velocities. Those parameters as well as the column density of each species are free to vary. The local continuum around the lines of interest was approximated by a polynomial of degree 0 to 3 . The continuum level and shape are also free parameters. The LSF width (Sect. 2) has little influence on the column density determination. This is principally due to the fact that the lines providing constraints on the column density are weak. The column density determination of all the species considered in this study varies by less than 0.1 dex when forcing the LSF width to vary between $20 \mathrm{~km} \mathrm{~s}^{-1}$ and $45 \mathrm{~km} \mathrm{~s}^{-1}$. Other LSF widths result in unsatisfactory fits based on the $\chi^{2}$ value. Lines were fitted simultaneously for the different observing channels to benefit from the data redundancy allowed by FUSE. The fitted lines are those in Table 2. Final fits are presented in Figs. 3-5 while derived column densities are given in Table 3. For consistency, we also derived column densities of metals using the spectrum corrected for stellar absorption (Sect. 4.2)

\subsubsection{Presence of saturated unresolved components}

In addition to the simple case of a single line of sight intersecting a neutral cloud with uniform properties (Sect. 4.1.1), we also consider the case of a collection of diffuse clouds producing multiple unresolved absorption components. Such clouds can have different physical conditions and chemical properties. It is commonly accepted that a complex absorption structure along a single line of sight usually does not introduce systematic errors on the column density determination (Jenkins 1996). However, the presence of multiple lines of sight inevitably complicates the analysis. The most obvious effect is the wavelength smearing due to the distribution of the lines of sight across the aperture (Sect. 2).

The unusually weak interstellar lines in Pox 36 may indicate that unresolved absorption components - if present - are not saturated. In such a case, the equivalent width of the integrated absorption line is simply the sum of the equivalent widths of the individual components, although the contribution from lines of sight toward stars with different UV brightnesses might not scale linearly (Labasque \& Boissé, in preparation). We check for the presence of possible saturated components by following the method described in Lebouteiller et al. (2006). We derive the maximum column density the narrow component $\left(b=2 \mathrm{~km} \mathrm{~s}^{-1}\right)$ in the center of a line can have before becoming saturated. The resulting values are given in Table 2 and should be compared to the column densities inferred from the global profiles (Table 3). It shows that $\mathrm{O}$ I and Si II lines could be strongly saturated if narrow components are present, that the Fe II line could be saturated, but that N I, P II, and Ar I lines are only close to saturation. Note that this represents only the worst-case scenario since intervening clouds might have in fact larger velocity dispersion. Nevertheless, these results emphasize the reliability of the $\mathrm{N} \mathrm{I}$, P II, and Ar I column density determinations.

\subsection{Hydrogen}

The interstellar $\mathrm{H}$ I lines are strongly contaminated by absorption from stellar atmospheres (Sect. 3.3). This can be best seen for the high-order lines of the Lyman series in Fig. 2 which display typical "V-wings". The derivation of the interstellar H I column densities is done in three steps:

- estimate the stellar H I absorption using the high-order Lyman lines (e.g., Ly $\epsilon$ ) whose wings are purely stellar in origin;

- correct Ly $\beta$ (whose wings are partly stellar and interstellar) by subtracting the stellar $\mathrm{H}$ I absorption;

- estimate the interstellar H I profile using the corrected Ly $\beta$ line.

We used the TLUSTY FUV absorption model spectra for O and B stars of Lanz \& Hubeny $(2003,2007)$ to reproduce the spectral continuum of Pox 36 . We chose to model the stellar spectrum of Pox 36 with a single stellar population, which we will show in Sect. 4.2.1, is a safe assumption. TLUSTY models are available for several temperatures and metallicities. We considered only main-sequence stars $(\log g=4.0)$ and have included the effects of stellar rotation. The average rotational velocities have been taken from Kippenhahn \& Weigert (1990) and Lang (1992). Finally, it must be noted that the velocity dispersion of stars within Pox 36, or between individual H II regions, is negligible before the stellar rotation velocity. To compare with the observed spectra, we normalized the latter using a spline fit for the continuum, and the synthetic spectra using the featureless continuum provided by TLUSTY.

\subsubsection{Stellar temperature}

The wings of the stellar $\mathrm{H}$ I lines are strongly dependent on temperature. The best constraint is given by the high-order lines of the Lyman series since the interstellar contribution in these lines is saturated and they have no damping wings. Moreover, $\mathrm{H}$ I lines are not affected by metallicity so that they are not prone to the degeneracy between temperature and metallicity.

Given the redshift of Pox 36 and the resulting contamination by metallic lines from the Milky Way (see full spectrum in Fig. 2), Ly $\epsilon$ is the cleanest $\mathrm{H}$ I line for use to constrain the stellar temperature. Its line profile gives a temperature of $30000 \mathrm{~K}$ (Fig. 6), corresponding to a B0 stellar population. The uncertainty on this temperature determination is smaller than the steps allowed by the models $(<2500 \mathrm{~K})$. Are other stellar types present in large quantities in Pox 36 ? Hotter stars would contribute narrow $\mathrm{H}$ I absorption lines, not modifying appreciably the "V-shaped" wings of the high-order Lyman lines. As for the wings of the low-order $\operatorname{Ly} \beta$ line, they are dominated by interstellar absorption. Cooler stars would produce more prominent "V-wings" which are not seen in the profiles of the high-order Lyman lines. We conclude that the stellar populations responsible for the stellar H I profile are mainly composed of B0 stars, with a possible small amount of hotter stars. 

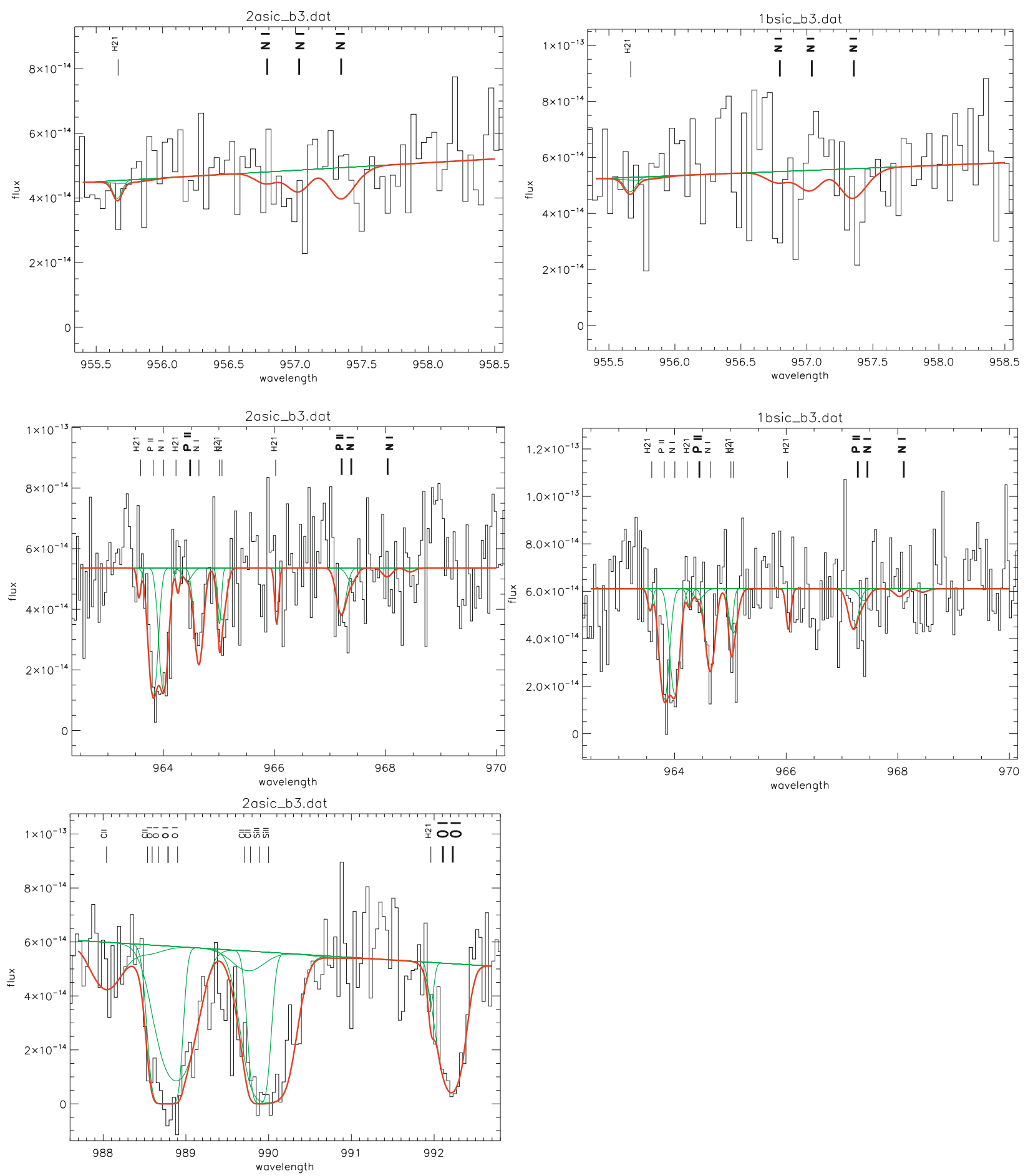

Fig. 3. Results of the profile fitting. The histogram shows the data. The thin profiles indicate individual absorption-lines while the thick profile indicates the total absorption. Lines labelled in bold arise in Pox 36 while the others arise in the Milky Way. Wavelength is expressed in $\AA$, while flux is expressed in $\operatorname{erg~s}^{-1} \mathrm{~cm}^{-2} \AA^{-1}$.

Based on the surface flux given by the models and assuming a distance of $20 \mathrm{Mpc}$ (Table 1), the observed FUV flux of Pox 36 implies that the equivalent number of $\mathrm{B} 0$ stars is $\sim 300$. This is the number of unextincted foreground stars.

\subsubsection{Stellar metallicity}

Lines from ionized metals (C III-IV, N III-V, Si IV-V, ...) arise in the stellar atmospheres of $\mathrm{O}$ and $\mathrm{B}$ stars. These lines can also contaminate the interstellar spectrum although their effect is much weaker as compared to the stellar H I lines. One expects that the metallicity of young stars to be similar to that of the H II gas (see e.g., Venn et al. 2001; Lee et al. 2006), being equal to $\sim 1 / 5 Z_{\odot}$ (Table 1 ) in the case of Pox 36 .

We computed TLUSTY models for a range of metallicities so they can be used to compare with observations. We use the prominent $\mathrm{C}$ III lines at $977.02 \AA$ and at $1175.6 \AA$ to derive an estimate of the stellar metallicity. The $977.02 \AA$ line is a 

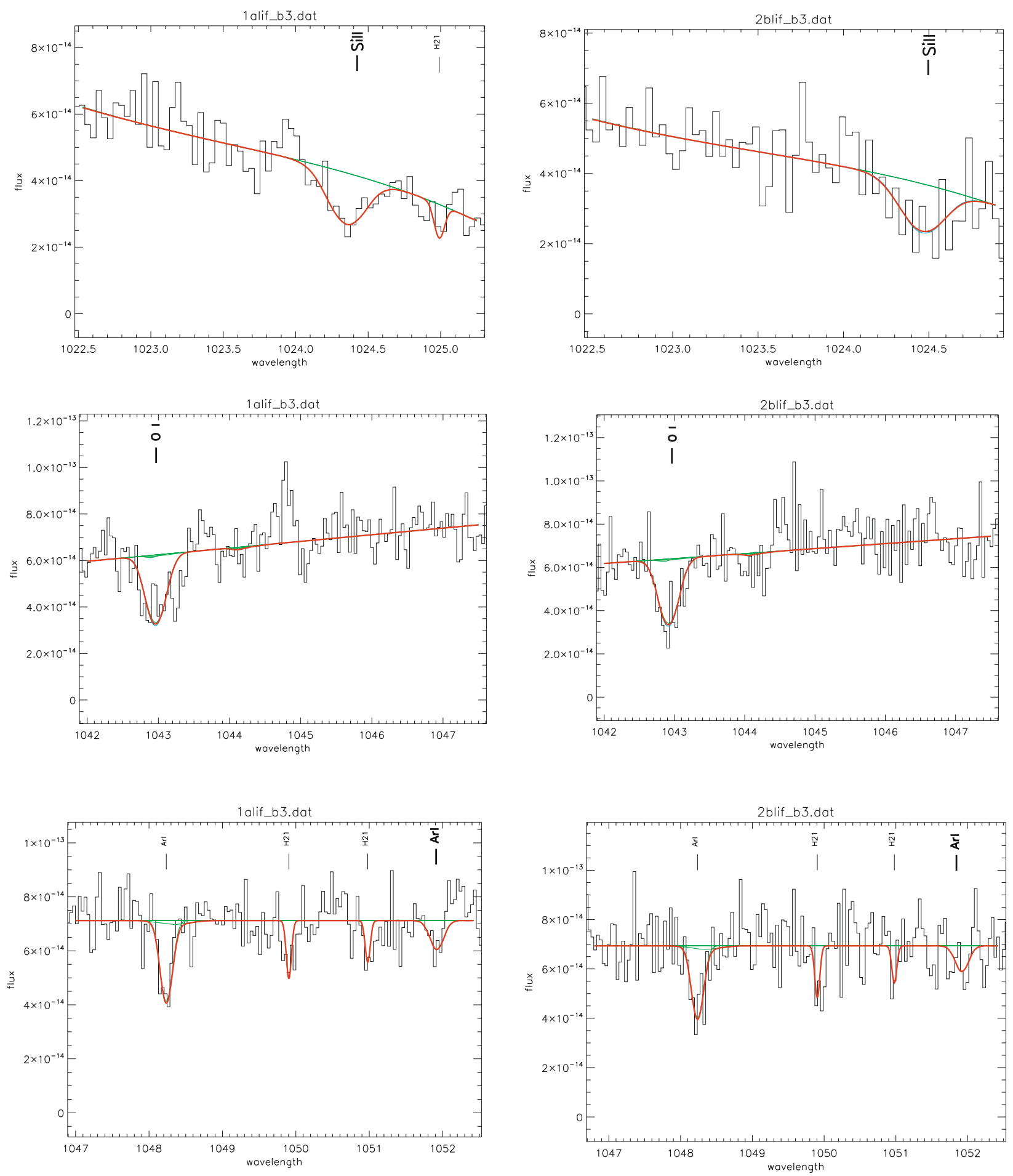

Fig. 4. See Fig. 3 for the plot description.

resonance line which can be either interstellar or stellar. It gives an upper limit to the stellar absorption. On the other hand, the $1175.6 \AA$ multiplet is not resonant and is purely stellar. Based on the two $\mathrm{C}$ III lines, we find that the observations agree best with a metallicity of $1 / 1000 Z_{\odot}$ (Fig. 7). This model fails however to reproduce the stellar absorption lines between $1110 \AA$ and $1120 \AA$ (Fig. 8). Inversely, the $1 / 5 Z_{\odot}$ model reproduces some stellar lines while overestimating other lines (notably around $\sim 1145 \AA$ ).

Thus it seems difficult to estimate the stellar metallicity by comparing the observations with the stellar lines predicted by models. This is of little importance for the interstellar
H I column density determination, but it may prevent a reliable estimate of the continuum for measuring metal column densities. In practice, the stellar lines are broad enough that they don't affect significantly the profiles of the narrow interstellar lines. We decided to use the $1 / 5 Z_{\odot}$ TLUSTY model and to rederive column densities of the metals using the normalized stellar absorption-corrected spectrum. The results are given in the last column of Table 3. They are consistent within the errors with those derived from spectra without correction for stellar metallic absorption, with perhaps the exception of the N I column density. Part of the small difference is probably due to systematic errors 

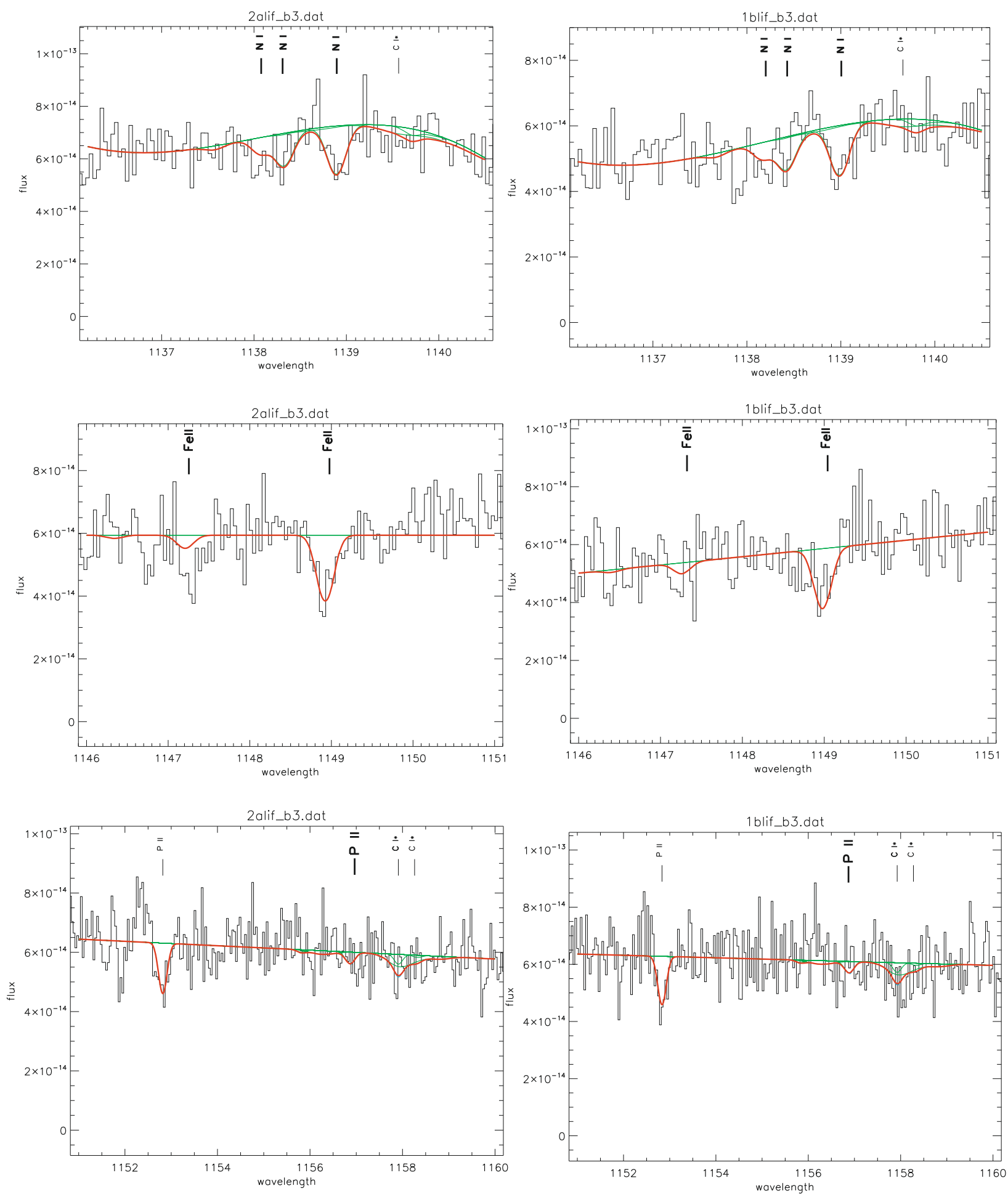

Fig. 5. See Fig. 3 for the plot description.

introduced in the normalization of the observations. Because the two determinations agree relatively well, we decide to use from now on the metal column densities derived from the observations without correction for stellar metallic absorption (Sect. 5).

\subsubsection{Interstellar Hı column density}

The interstellar H I content is mostly constrained by the damping wings of the $\operatorname{Ly} \beta$ line, since higher-order lines are saturated. In order to correct for the stellar absorption, we use the
TLUSTY stellar model. We chose a metallicity of $1 / 1000 Z_{\odot}$ instead of $1 / 5 Z_{\odot}$ to avoid uncertainties on the metallic stellar lines which we already saw cannot reproduce well the observations (Sect. 4.2.2). This is a safe choice for deriving interstellar $\mathrm{H}$ I column density since the $\mathrm{H} \mathrm{I}$ line profiles are independent of metallicity. In practice, our results on the $\mathrm{H}$ I column density do not depend on the metallicity chosen for the stellar models.

We thus built the interstellar spectrum by removing the stellar contribution. In Fig. 9, the observations are compared to synthetic profiles taking into account stellar and interstellar 
Table 3. Column densities. Errors are given at $2 \sigma$.

\begin{tabular}{lll}
\hline \hline Species & $\begin{array}{l}N_{\text {obs }}^{a} \\
\left(\mathrm{~cm}^{-2}\right)\end{array}$ & $\begin{array}{l}N_{\text {norm }}^{b} \\
\left(\mathrm{~cm}^{-2}\right)\end{array}$ \\
\hline N I & $14.20_{-0.14}^{+0.12}$ & $13.96_{-0.33}^{+0.22}$ \\
O I & $15.58_{-0.09}^{+0.12}$ & $15.52_{-0.09}^{+0.10}$ \\
Si II & $15.06_{-0.28}^{+0.19}$ & $15.00_{-0.13}^{+0.13}$ \\
P II & $12.78_{-0.32}^{+0.22}$ & $12.94_{-0.21}^{+0.16}$ \\
Ar I & $13.18_{-0.29}^{+0.19}$ & $13.10_{-0.79}^{+0.31}$ \\
Fe II & $13.98_{-0.13}^{+0.11}$ & $13.97_{-0.18}^{+0.13}$ \\
\hline
\end{tabular}

${ }^{a} N_{\text {obs }}$ is the column density calculated using local spectral continuum from the observation.

${ }^{b} N_{\text {norm }}$ is the column density calculated using the spectrum corrected from stellar absorption (Sect. 4.2).

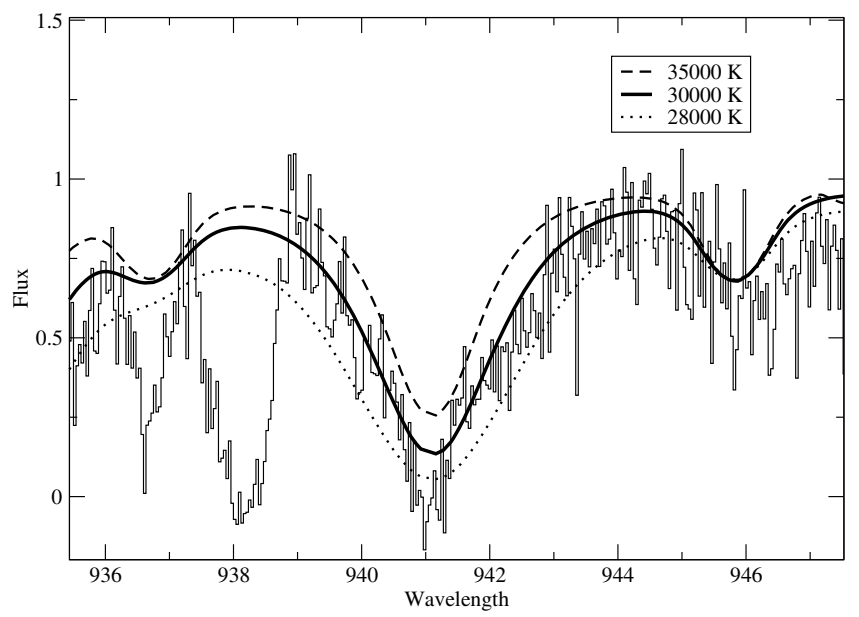

Fig. 6. The Ly $\epsilon$ line profile gives a strong constraint on the photosphere temperature of the dominant stellar population. Models are drawn for a metallicity of $1 / 1000 Z_{\odot}$, but the profile of stellar $\mathrm{H}$ I lines is unchanged for varying metallicities.

absorptions. More general cases with various stellar temperatures and lines other than $\operatorname{Ly} \beta$ can be found in the Appendix A. The best model agrees with $\log N(\mathrm{H} \mathrm{I}) \approx 20.3$. The final fit, after removal of the stellar absorption is shown in Fig. 10. The most likely column density is $\log N(\mathrm{H} \mathrm{I})=20.28 \pm 0.06$. The uncertainty is only statistical and does not include systematic errors on the stellar model. We studied the influence of the stellar model on the H I column density by considering models at $29000 \mathrm{~K}$ and $32500 \mathrm{~K}$. The fits can be found in Fig. 10. The H I column density for the $29000 \mathrm{~K}$ models is $20.18 \pm 0.08$. For such a temperature, even Ly $\beta$ 's profile is almost independent of the interstellar contribution. In fact, the interstellar $\mathrm{H}$ I profile for $28000 \mathrm{~K}$ is already saturated without any damping wings, and the $\mathrm{H}$ I column density is not constrained $(\log N(\mathrm{H} \mathrm{I})<20.1)$. For $32500 \mathrm{~K}$, the $\mathrm{H}$ I column density is $20.66 \pm 0.06$. In that case, the higher temperature results in narrow stellar lines, and the interstellar contribution, and damping wings are clearly seen. Since a temperature of $32500 \mathrm{~K}$ is clearly too high to reproduce correctly high-order Lyman lines (Sect. 4.2.1), we consider that the corresponding $\mathrm{H}$ I column density should be regarded as a conservative upper limit. It must be noted that the fit quality (given by the $\chi^{2}$ ) is almost identical for the 3 temperatures we tested. The reason is that $\mathrm{Ly} \beta$ is not a strong constraint on the stellar temperature contrary to Ly $\epsilon$ (Sect. 4.2.1). We use hereafter the interstellar H I column density $\log N(\mathrm{H} \mathrm{I})=20.3 \pm 0.4$ with conservative
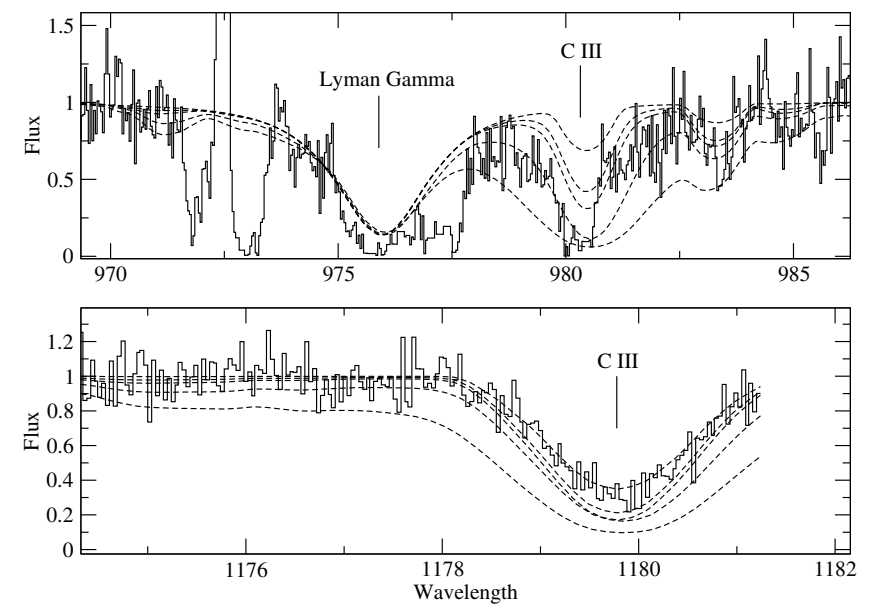

Fig. 7. The profile of the C III lines at $977.02 \AA$ (top) and at $1175.6 \AA$ (bottom) is strongly affected by the metallicity. The models are from bottom to top for $Z=1 / 2,1 / 10,1 / 50,1 / 100$, and $1 / 1000 Z_{\odot}$.

error bars which include uncertainties on the best stellar model, on the stellar model itself, and on the interstellar profile fitting.

Is this determination in agreement with radio observations? Assuming the neutral gas to be optically thin and a distance of $20 \mathrm{Mpc}$, the integrated $\mathrm{H}$ I radio flux (Table 1) gives a mass of $M(\mathrm{HI})=8.1 \times 10^{8} M_{\odot}$. If the $\mathrm{H}$ I distribution is uniform, the column density we derived implies that the $\mathrm{H}$ I diameter is $13 \mathrm{kpc}$. Based on the image of Fig. 1, the angular diameter of the galaxy in the optical is close to $30^{\prime \prime}$, which at the distance of Pox 36 corresponds to $\approx 3 \mathrm{kpc}$, i.e., a factor $\approx 4$ smaller than the $\mathrm{H}$ I extent. This is consistent with H I observations of BCDs (e.g., Thuan \& Martin 1981). We conclude that the H I column density measured in the FUV is in agreement with radio observations.

\section{Chemical abundances}

In this section we derive the chemical abundances from the column densities. Abundance determinations are mostly affected by ionization corrections and by element depletion on dust grains.

\subsection{Ionization structure}

The abundance of an element is usually estimated from its primary ionization state and by applying ionization correction factors. We expect that all elements with ionization potentials larger than that of hydrogen $(13.6 \mathrm{eV})$ are in a neutral state in the $\mathrm{H}$ I gas. This is the case for N, O, and Ar. On the other hand, $\mathrm{Si}, \mathrm{P}$, and Fe are mostly found as single-charged ions with negligible fractions of neutral atoms.

Some nitrogen and argon could be ionized in places where hydrogen is neutral. This is a consequence of the large photoionization cross-section of $\mathrm{N} \mathrm{I}$ and Ar I together with the penetration of UV photons in low-density partly-ionized regions of the ISM (Sofia \& Jenkins 1998). We expect the ionization correction factor for N I to be much smaller than for Ar I. Our results suggest in fact that they are both negligible (Sect. 6).

Inversely, ionization corrections for $\mathrm{Si}, \mathrm{P}$, and $\mathrm{Fe}$ may be needed if the singly-ionized ions exist in the ionized gas of $\mathrm{H}$ II regions and contribute to the observed FUV absorption lines. For the giant H II region NGC 604, Lebouteiller et al. (2006) found that only Si II and P II require significant ionization corrections ( 0.34 dex and 0.15 dex resp.; see also Aloisi et al. 2003 in IZw 18). Thuan et al. (2002) estimated the amount of Fe II in 

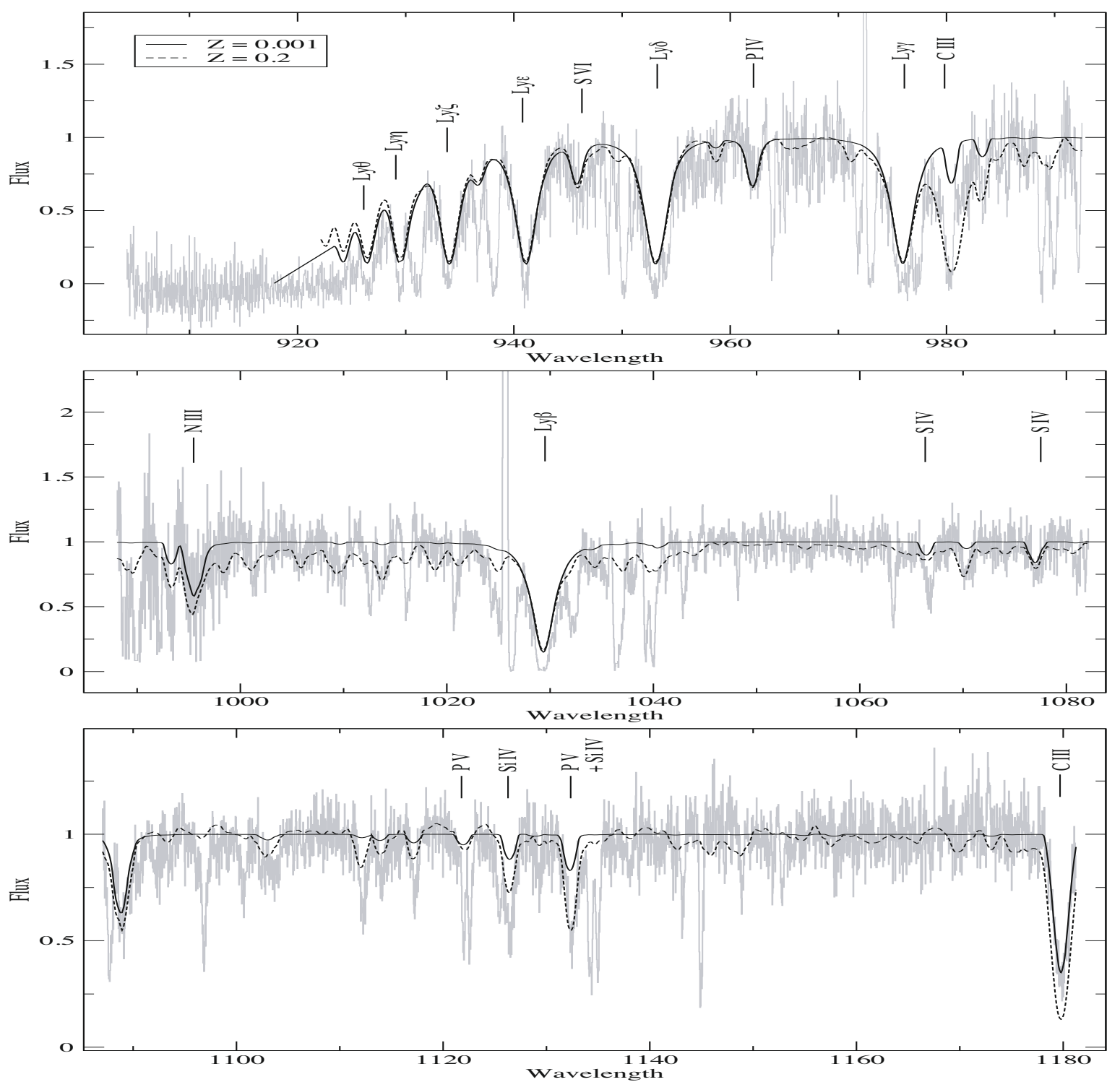

Fig. 8. The stellar models for $Z=1 / 1000 Z_{\odot}$ (solid line) and $1 / 5 Z_{\odot}$ (dashed line) are superimposed on the normalized observed spectrum (gray histogram). The most prominent stellar lines are labelled.

the ionized gas of Mark 59 to be $20 \%$ of the total iron abundance derived from the absorption-lines. Therefore, we could expect ionization corrections on the same order to exist in Pox 36. We neglect them in the following discussion because it has only little influence on our results.

We finally use N I, O I, Si II, P II, Ar I, and Fe II to derive chemical abundances (Table 4 ). It must be reminded that the $\mathrm{H}$ I column density is larger than the metal column densities by several orders of magnitude, so that weak absorption components could contribute to the damped H I line $\operatorname{Ly} \beta$ while the corresponding component in metal lines are below the detection limit (see Hébrard et al. 2003 for the effect in D/H determinations). This would lower the interstellar abundance determinations as compared to their true values (Sect. 6).

\subsection{Depletion on dust grains}

The refractory elements $\mathrm{Si}$ and Fe are likely to be locked on dust grains and thus depleted from the diffuse phase. To compare the abundances of the neutral and ionized gas, we need to take into account potential depletion effects.

It is difficult to assess the depletion of elements in the ionized gas of Pox 36 since only one refractory element, Fe, has been observed in this phase. The use of iron as a probe of depletion is complicated by the fact that it might be released in the ISM on larger timescales. In the Milky Way, it is thought that about $2 / 3$ of the iron in the ISM was produced by SNe type Ia involving low-mass stars. Rodriguez \& Esteban (2004) and Izotov et al. (2006) observed that the $[\mathrm{O} / \mathrm{Fe}]$ ratio increases with metallicity in emission-line galaxies, being consistent with depletion of $\mathrm{Fe}$ on dust grains, and with iron production in $\mathrm{SN}$ type II in those galaxies. The $[\mathrm{O} / \mathrm{Fe}]$ ratio in the ionized gas of Pox 36 is $[\mathrm{O} / \mathrm{Fe}]_{i}=1.12 \pm 0.12$, i.e., much larger than the mean value of $0.40 \pm 0.14$ in the ionized gas of BCDs (Izotov \& Thuan 1999). Accordingly, this should imply a significant iron depletion on dust grains in this phase.

The dust content in the neutral gas of BCDs is unfortunately largely unknown. FUV observations only select lines of sight with little dust content. Vidal-Madjar et al. (2000) have explained the absence of diffuse $\mathrm{H}_{2}$ in the BCD IZw18 by the low 


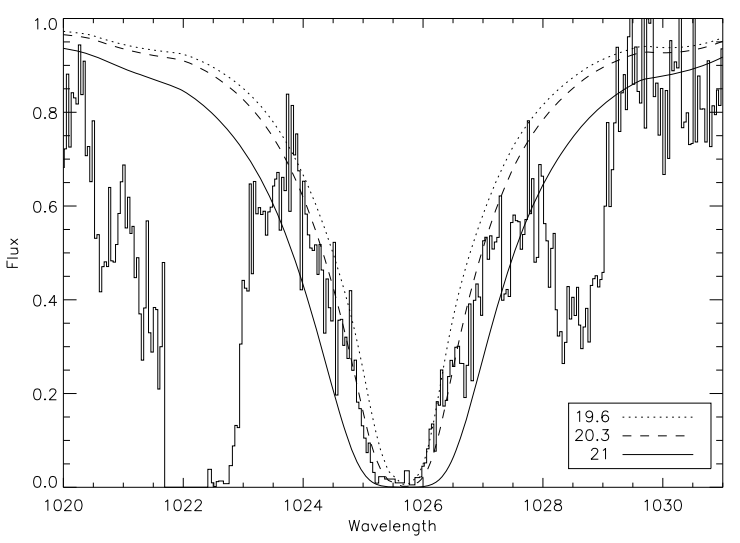

Fig. 9. The observed $\mathrm{Ly} \beta$ profile is compared to synthetic profiles combining the stellar model $(30000 \mathrm{~K})$ and interstellar H I profiles with various column densities.

abundance of dust grains (together with the low H I volume density and the hard radiation field). To date, no diffuse $\mathrm{H}_{2}$ has been detected in other BCDs, including Pox 36 (Sect. 3.1). Therefore, we expect $\mathrm{Fe}$ to be less depleted in the neutral gas of Pox 36 than in the ionized gas. This is the case: the $[\mathrm{O} / \mathrm{Fe}]_{\mathrm{n}}$ in the neutral gas of Pox 36 is $0.39 \pm 0.16$, much lower than the value in the ionized gas $(1.12 \pm 0.12)$, but consistent with the mean value in the ionized gas of BCDs (Izotov \& Thuan 1999). We conclude that iron is nearly not depleted on dust grains in the neutral gas of Pox 36. Furthermore, since silicon is usually less depleted than iron (e.g., Sofia et al. 1994; Savage \& Sembach 1996), our silicon abundance determination should also be little affected by dust depletion.

\section{Results}

Based on the metal abundances of Table 4, it is clear that the neutral gas in Pox 36 is not pristine: it has already been enriched with heavy elements. This is of course expected since Pox 36 is more metal-rich than IZw 18 and SBS 0335-052 where no pristine gas has been found either (Aloisi et al. 2003; Lecavelier et al. 2004; Thuan et al. 2005). The origin of metals in the neutral phase is however unclear. The presence of old stellar population in the great majority of BCDs (e.g., Aloisi et al. 1999; Östlin 2000) leads to the conclusion that previous enrichment could have occured in previous starburst episodes or through low-level star-formation over the galaxy's history. Comparison with the chemical composition of the ionized gas in the $\mathrm{H}$ II regions provides an interesting diagnostic to understand the enrichment process of the neutral gas.

\subsection{Comparison with the ionized gas of the HII regions}

Table 4 shows that $\mathrm{N}$ is underabundant by a factor $\approx 4$ in the neutral gas as compared to the ionized gas, while both $\mathrm{O}$ and $\mathrm{Ar}$ are underabundant by a factor $\approx 6$ and $\approx 8$ respectively. The relatively good agreement between the deficiency factors of $\mathrm{N}, \mathrm{O}$, and $\mathrm{Ar}$ in the neutral gas indicates that the abundances of $\mathrm{N}$ and Ar are probably well determined (Sect. 5.1). The iron abundance appears to behave differently, being the same within the errors in the two gaseous phases. However, if we correct $[\mathrm{O} / \mathrm{Fe}]$ in the ionized gas of Pox 36 for dust depletion (Sect. 5.2), the iron abundance becomes:

$[\mathrm{Fe} / \mathrm{H}]_{i}^{*} \sim[\mathrm{Fe} / \mathrm{H}]_{i}+[\mathrm{O} / \mathrm{Fe}]_{i}-0.40$,
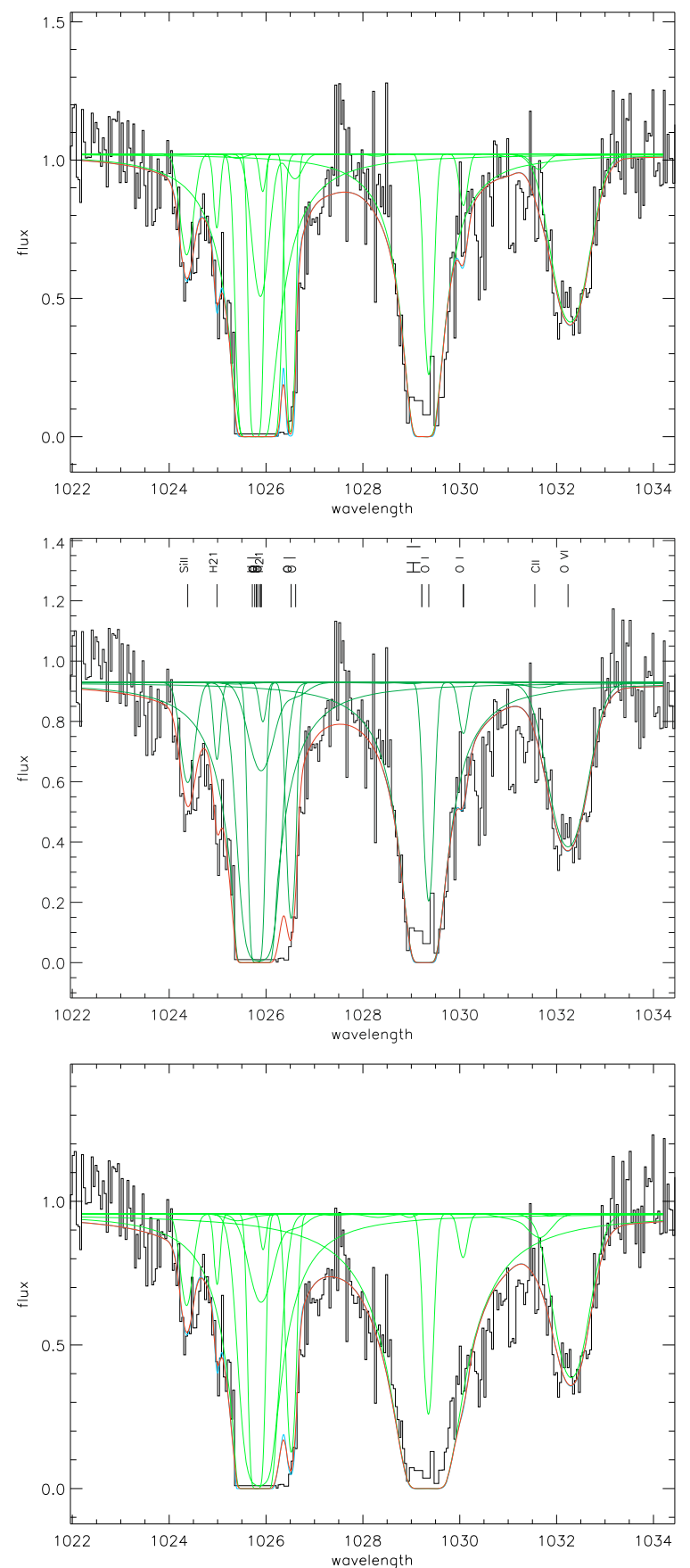

Fig. 10. Profile fitting of $\operatorname{Ly} \beta$ in Pox 36 at the observed wavelength of $\approx 1029.4 \AA$. The spectra are corrected for stellar absorption using models at $29000 \mathrm{~K}$ (top), $30000 \mathrm{~K}$ (middle), and $32500 \mathrm{~K}$ (bottom). The corresponding line in the Milky Way can be seen at the nominal wavelength of $\approx 1025.72 \AA$.

giving $[\mathrm{Fe} / \mathrm{H}]_{i}^{*} \sim-0.9$, about 7 times higher than the value in the neutral gas. Thus $\mathrm{Fe}$ is underabundant in the neutral gas by the same factor as N, O, and Ar.

Can this metal deficiency in the neutral gas be attributed to an overestimate of the $\mathrm{H}$ I column density? Considering the metal column densities of Table 3, the neutral gas would have the same metallicity as the ionized gas if the $\mathrm{H}$ I column density is on the order of $\log N(\mathrm{H} \mathrm{I}) \approx 19.6$. This is inconsistent with the observations since such a low column density does not reproduce the wings of $\operatorname{Ly} \beta$ (Fig. 9). We conclude that the discontinuity 
Table 4. Chemical abundances. $[\mathrm{X} / \mathrm{H}]$ is defined as $\log (\mathrm{X} / \mathrm{H})-$ $\log (\mathrm{X} / \mathrm{H})_{\odot}$, where $\log (\mathrm{X} / \mathrm{H})_{\odot}$ is the solar abundance. Solar abundances are from Asplund et al. (2005).

\begin{tabular}{llll}
\hline \hline Element (tracer) & $\log (\mathrm{X} / \mathrm{H})$ & {$[\mathrm{X} / \mathrm{H}]_{\mathrm{n}}$} & {$[\mathrm{X} / \mathrm{H}]_{i}{ }^{b}$} \\
\hline $\mathrm{N}(\mathrm{N}$ I) & $-6.10 \pm 0.42$ & $-1.88 \pm 0.42$ & $-1.31 \pm 0.06$ \\
$\mathrm{O}(\mathrm{O}$ I) & $-4.72 \pm 0.41$ & $-1.38 \pm 0.41$ & $-0.61 \pm 0.05$ \\
$\mathrm{Si}(\mathrm{Si}$ II $)$ & $-5.24 \pm 0.47$ & $-0.75 \pm 0.47$ & \\
$\mathrm{P}(\mathrm{P}$ II $)$ & $-7.52 \pm 0.47$ & $-0.88 \pm 0.47$ & \\
$\mathrm{Ar}(\mathrm{Ar}$ I) & $-7.12 \pm 0.47$ & $-1.30 \pm 0.47$ & $-0.42 \pm 0.06$ \\
$\mathrm{Fe}(\mathrm{Fe}$ II $)$ & $-6.32 \pm 0.42$ & $-1.77 \pm 0.42$ & $-1.62 \pm 0.11$ \\
\hline
\end{tabular}

${ }^{a}$ Abundance in the neutral gas (this study).

${ }^{b}$ Abundance in the ionized gas (Izotov \& Thuan 2004).

between the metallicity of the neutral and ionized gas is not driven by uncertainties on the H I column density determination.

Oxygen and argon are the best metallicity tracers available in the ionized gas, and agree with a metallicity of $\approx 1 / 5 Z_{\odot}$ in this phase. Considering these 2 elements, we find that the metallicity in the neutral gas is $\approx 1 / 35 Z_{\odot}$, i.e., a factor $\sim 7$ below the value in the ionized gas. Our results for Pox 36 are similar to those obtained for other BCDs studied by FUSE (Sect. 7): although the neutral gas of Pox 36 is not pristine, it is less chemically evolved than the ionized gas in the $\mathrm{H}$ II regions.

\subsection{Pristine gas pockets}

It is possible that pockets of pure $\mathrm{H}$ I lie in the halo, while only a fraction of the $\mathrm{H}$ I has been enriched close to the starburst region. This would dilute the chemical abundances inferred from integrated lines of sight by adding $\mathrm{H}$ I without any metallic counterpart. In Pox 36, the $\mathrm{H}$ I has a radial velocity slightly lower than that of the metals (Sect. 3.1). The same result was observed in IZw18 by Aloisi et al. (2003) where the H I has a velocity of $753 \pm 6 \mathrm{~km} \mathrm{~s}^{-1}$ as compared to $765 \pm 10 \mathrm{~km} \mathrm{~s}^{-1}$ for the metals. If the offset is real (i.e., not driven by systematic uncertainties on the wavelength calibration), this suggests that part of the $\mathrm{H}$ I could lie indeed in regions without any metals. This would imply that the ISM of the galaxy is in fact not well mixed, with much lower abundances toward the external regions. The offset is however too small to assert unambiguously the presence of metal-free gas.

\subsection{Enrichment process}

We now calculate the amount of metals produced and released by the present starburst episode in order to understand the metal enrichment of the neutral gas. The stellar mass formed is derived from the UV luminosity, following Lequeux et al. (1981). We assume an initial mass function slope of -1.5 and a burst age of $10 \mathrm{Myr}$ (see Sect.3.3). We find that the stellar mass is $2.5 \times 10^{6} M_{\odot}$. Yields were taken from Meynet \& Maeder (2002), assuming a metallicity of $0.2 Z_{\odot}$ and a mass interval of $[2-120] M_{\odot}$. We find that an oxygen mass of $M(\mathrm{O})=1.25 \times$ $10^{4} M_{\odot}$ is eventually released by the starburst. Several scenarios are then possible, depending on the volume extent over which the dispersal and the mixing occurs.

We first assume that all the newly produced oxygen mixes with the ionized gas. Following Kunth \& Sargent (1986), the mass of ionized gas is:

$M(\mathrm{HII})=\frac{N m_{\mathrm{p}}}{n_{i} \beta}$, where $N$ is the number of ionizing photons, $m_{\mathrm{p}}$ is the proton mass, $n_{i}$ is the electronic and ionic density, and $\beta$ is the recombination coefficient. From the $\mathrm{H} \beta$ luminosity (Table 1 ), we find $N=4.4 \times 10^{50} \mathrm{~s}^{-1}$. We calculate $\beta=2.482 \times 10^{-14} \mathrm{~cm}^{3} \mathrm{~s}^{-1}$ from the table of Hummer \& Storey (1987) using the electron density $\left(60 \mathrm{~cm}^{-3}\right)$ and temperature $(12560 \mathrm{~K})$ found by Izotov et al. (2004). The mass of ionized gas is then $M(\mathrm{HII})=2.5 \times 10^{5} M_{\odot}$. If the SNe products mix only with the ionized gas, the metallicity of this phase would reach an additional oxygen enrichment of $12+\log (\mathrm{O} / \mathrm{H})=9.64$. This unrealistic value suggests that heavy elements will mix with a larger mass of gas, at larger spatial scales.

Next, we assume that the newly produced oxygen will mix uniformly with the $\mathrm{H}$ I gas. Using the H I mass from Table 1, we find that the neutral gas would be enriched by an additional $5 \%$ only $(12+\log (\mathrm{O} / \mathrm{H})=5.98)$. Therefore, it can be seen that many starburst episodes $(\sim 20)$ are required for the neutral gas to reach the metallicity level we derived with FUSE.

A possible alternative is that the mixing of new metals might not be uniform as the $\mathrm{H}$ I lying close to the stellar clusters could be more enriched than the outskirts which would remain metalfree. In order for a nearby $\mathrm{H}$ I gas shell to have the same metallicity as that of the star-forming region (ionized gas), we find that the newly produced oxygen should mix with $7.0 \times 10^{7} M_{\odot}$ of $\mathrm{H}$ I, i.e., $\sim 10 \%$ of the total $\mathrm{H}$ I in the galaxy. This can be seen as the upper limit of the H I volume that can be enriched to reasonable levels. We conclude that SNe products could mix with a small fraction of the H I gas in Pox 36 while not enriching most of it. This hypothesis implies that pockets of pristine gas could well be present in the galaxy. One way to prove this is to study absorption-lines toward several star-forming regions of a given galaxy, with significantly different foreground gas content. Another way is to analyze the scatter of the relation between abundances in the ionized gas and neutral gas for a sample of BCDs (Sect. 7.1).

Toward a plausible scenario, it could thus be that the vast majority of the metals released during a starburst episode mixes with a large mass of $\mathrm{H} \mathrm{I}$, increasing only slightly its metallicity, while a small fraction mixes with the star-forming region itself (currently ionized), increasing greatly its metallicity. These metals most likely come from previous episodes of star-formation, with the consequence that star-formation episodes have to be cospatial. We consider the simple case in which the metallicity of the neutral phase increases by $f_{\mathrm{n}} m / M(\mathrm{HI})$, where $m$ is the mass of metals released by a starburst and $f_{\mathrm{n}}$ is the fraction of these metals to be mixed within the H I region. Similarly, the ionized gas increases its metallicity by $f_{i} m / M(\mathrm{HII})$. Over successive starburst episodes, a metallicity offset between the ionized and neutral phases is achieved and maintained if the following condition is met:

$f_{\mathrm{n}}=\frac{1}{1+\alpha \frac{M(\mathrm{HII})}{M(\mathrm{HI})}}$,

where $\alpha$ is a parameter depending on the metallicity offset. An offset of $1 \mathrm{dex}-$ as it is observed in BCDs - implies $\alpha=10$. If, as in Pox 36, the H I mass is 1000 times larger than the ionized gas mass, the fraction of metals mixing with the $\mathrm{H}$ I phase should be $\approx 99 \%$, while only $1 \%$ mixes with the gas of the star-forming region. In conclusion, under the previous condition, the neutral gas can remain metal-poor, compared to the ionized gas, as long as the star formation keeps occurring at the same location. If another burst occurs at another location, one might expect to measure low abundances, but the offset will reappear when the next starburst episode occurs and after mixing is achieved. 

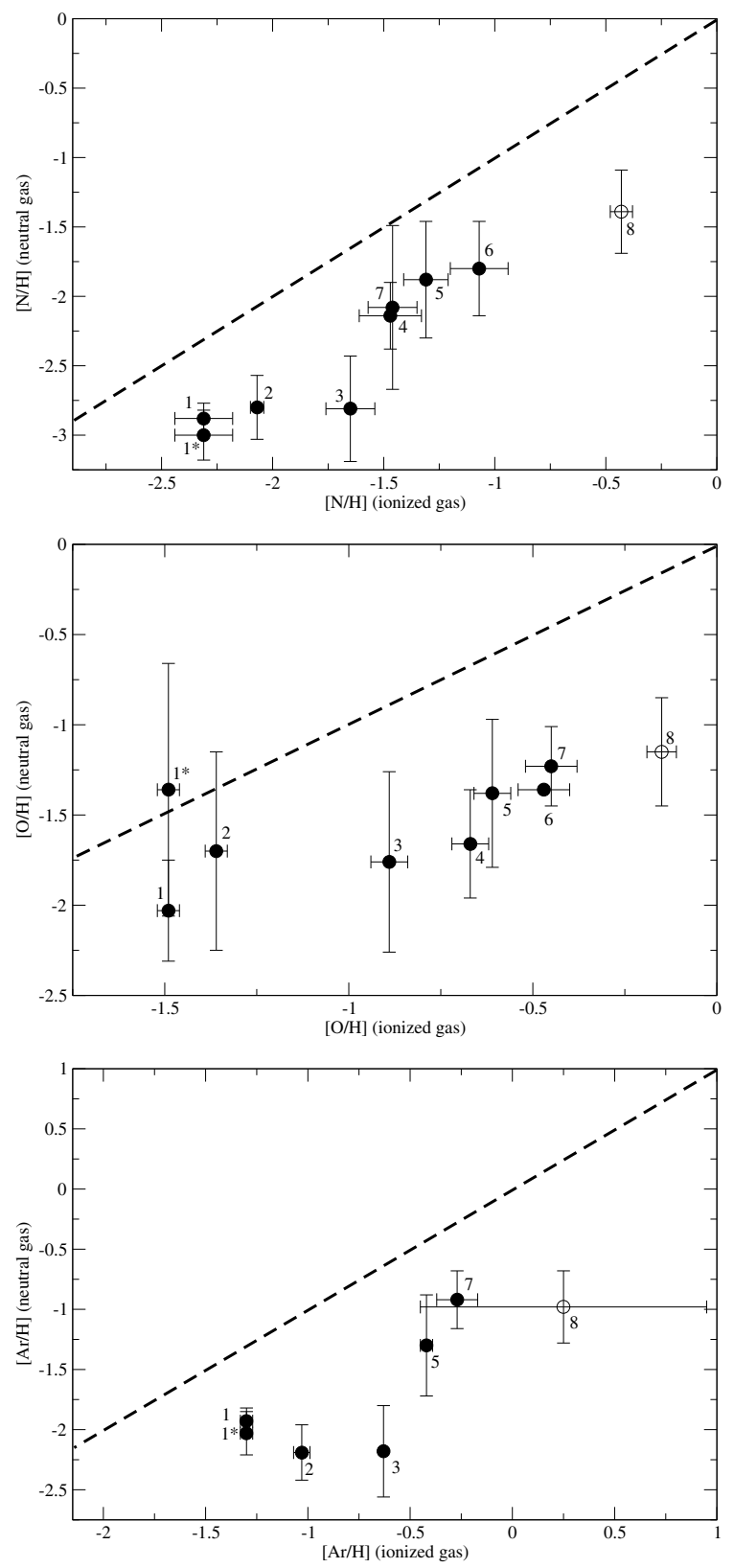

Fig. 11. The abundances of nitrogen (top), oxygen (middle), and argon (bottom) in the ionized gas and in the neutral gas are compared. The dashed line indicates the 1:1 ratio. Labels: (1*) IZw 18 (Lecavelier des Etangs et al. 2004), (1) IZw 18 (Aloisi et al. 2003), (2) SBS 0335-052 (Thuan et al. 2005), (3) IZw 36 (Lebouteiller et al. 2004), (4) Mark 59 (Thuan et al. 2002), (5) Pox 36 (this study), (6) NGC 625 (Cannon et al. 2005), (7) NGC 1705 (Heckman et al. 2001), (8) NGC 604/M 33 (Lebouteiller et al. 2006).

\section{Discussion}

\subsection{Chemical enrichment of BCDs}

The FUSE BCD sample is now large enough to allow the study of general trends. Are the results concerning Pox 36 consistent with those of other BCDs? A total of 7 BCDs with various metallicities $\left(1 / 50\right.$ to $\left.1 / 3 Z_{\odot}\right)$ have now been analyzed, in addition to the high-metallicity giant $\mathrm{H}$ II region NGC 604 in the spiral galaxy M33. We plot in Fig. 11 the elemental abundance

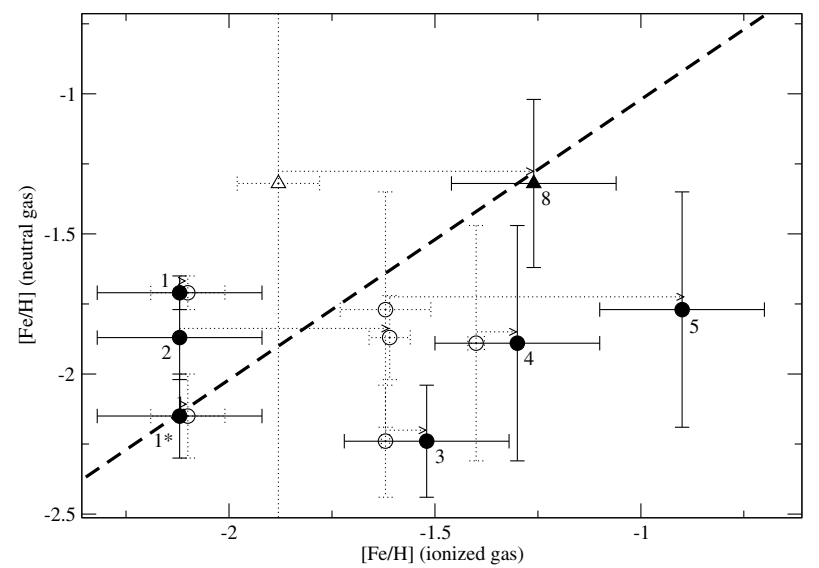

Fig. 12. Abundances of Fe in the neutral gas and in the ionized gas. Grey points correspond to the $[\mathrm{Fe} / \mathrm{H}]$ in the ionized gas while black dots include a correction to account for depletion (Sect. 6). See Fig. 11 for the labels.

$[\mathrm{X} / \mathrm{H}]^{3}$ in both the ionized and neutral gas of the BCDs. It can be seen that N, O, and Ar show identical trends, with systematically lower abundances in the neutral ISM than in the ionized gas. Part of the dispersion of $[\mathrm{O} / \mathrm{H}]$ is probably due to saturation effects which could not be avoided in some objects. Some of the dispersion of $[\mathrm{N} / \mathrm{H}]$ and $[\mathrm{Ar} / \mathrm{H}]$ is probably due to ionization corrections. Since the trend is similar for N, O, and Ar, the corrections are either of the same order for all objects ( $\leq 0.5 \mathrm{dex})$, or they are negligible, as for Pox 36 (Sect. 6). The abundance of iron in the neutral and ionized gas of BCDs must be interpreted with more care because of depletion on dust grains (Sect. 5.2). Figure 12 shows the relation between the Fe abundances in the two gaseous phases, considering both the observed abundances and the ones corrected for depletion. It can be seen that corrected values agree more with the general trends observed for $\mathrm{N}, \mathrm{O}$, and Ar (Fig. 11). It is seen in particular that iron depletion affects significantly the ionized gas abundances, especially in SBS 0335052, Pox 36, and NGC 604.

Examination of $[\mathrm{Ar} / \mathrm{H}]$ and $[\mathrm{N} / \mathrm{H}]$ in Fig. 11 reveals the possible existence of a plateau for the lowest-metallicity BCDs $\left(12+\log (\mathrm{O} / \mathrm{H})_{i} \leq 7.8\right.$ or $\left.\lesssim 1 / 8 Z_{\odot}\right)$. The data on $[\mathrm{O} / \mathrm{H}]_{\mathrm{n}}$ are also consistent with the existence of a metallicity plateau at $12+\log (\mathrm{O} / \mathrm{H})_{\mathrm{n}} \sim 7.0\left([\mathrm{O} / \mathrm{H}]_{\mathrm{n}}=-1.7\right)$, although the error bars are considerably larger. The presence of a plateau would imply a minimal enrichment of the neutral ISM. It could be that the $\mathrm{H}$ I reservoir has this minimal metallicity before star-formation ever occurred. Interestingly, Telfer et al. (2002) found that the intergalactic medium in the redshift range $1.6<z<2.9$ has a metallicity $12+\log (\mathrm{O} / \mathrm{H})$ between 6.7 and 7.6, which includes the floor metallicity observed in BCDs. Hence it appears that the neutral and ionized ISM of the lowest metallicity galaxies could well be characterized by a default enrichment rather than by previous star-formation episodes. In opposition, the highermetallicity objects would be enriched by many starburst episode. Further observations and analysis of BCDs with metallicities lower than $\sim 1 / 8 Z_{\odot}$ will be needed to confirm the existence of this plateau.

At higher metallicities $\gtrsim 1 / 5 Z_{\odot}$, the abundances of the neutral gas and ionized gas seem to be well correlated. This is the case for Mark 59, Pox 36, NGC 625, and NGC 1705. The giant $\mathrm{H}$ II region NGC 604 appears to extend the trend to nearly

\footnotetext{
${ }^{3}[\mathrm{X} / \mathrm{H}]$ is defined as $\log (\mathrm{X} / \mathrm{H})-\log (\mathrm{X} / \mathrm{H})_{\odot}$, where $\log (\mathrm{X} / \mathrm{H})_{\odot}$ is the solar abundance from Asplund et al. (2005).
} 
solar metallicities. The neutral gas abundances are systematically lower than those of the ionized gas. Nitrogen, oxygen, and argon in the neutral gas are underabundant by a factor $\sim 0.9 \mathrm{dex}$, i.e., nearly 10, as compared to the ionized gas.

If we dismiss the idea of a plateau and consider instead that the points in Fig. 11 show a positive correlation with significant scatter from the lowest to the highest metallicity objects, then the situation could be consistent with a dilution by metal-free gas along the lines of sight. The scatter would then be due to the dilution factor being different for each object. However one would then expect objects with the largest $\mathrm{H}$ I column density to display the largest underabundance in the neutral gas. We studied the dependences of the underabundance factor with the $\mathrm{H}$ I column density but found no significant correlation.

\subsection{Interpretation}

The lower metallicity observed in the $\mathrm{H}$ I regions of BCDs as compared to the ionized gas of their $\mathrm{H}$ II regions remains a puzzle. We have discussed many effects that may affect the determination of the neutral gas abundances such as the source extent, ionization corrections, depletion on dust grains, stellar contamination, and line saturation. The underabundance of a factor 10 found in the $\mathrm{H}$ I region of $\mathrm{BCDs}$ is however so dramatic that it seems difficult all these effects can make us miss the detection of $90 \%$ of the metals. Therefore, this offset appears to be real and we discuss in the following possible physical explanations. While self-enrichment of the H II regions, as proposed by Kunth \& Sargent (1986), naturally explains why metals are more abundant in the ionized gas, this scenario is difficult to reconcile with the homogeneous abundances observed in disconnected H II regions of most dwarf galaxies (e.g., Dufour 1986; Russell \& Dopita 1990; Skillman \& Kennicut 1993; Kobulnicky \& Skillman 1996; Kobulnicky \& Skillman 1997; Legrand et al. 2000; Noeske et al. 2000). In fact, the observed dispersion is probably dominated by variations of the electron temperature and not by intrinsic chemical inhomogeneities. Much less frequent is the observation of local discontinuities, with only NGC 5253 (Welch 1970; Walsh \& Roy 1989; Kobulnicky et al. 1997; López-Sánchez et al. 2007), IIZw 40 (Walsh \& Roy 1993), IC 4662 and ESO 245-G05 (Hidalgo-Gámez et al. 2001). Still, the local pollution could be generally explained by Wolf-Rayet winds (Brinchman et al. 2008) rather than by SNe explosions.

Based on the gas kinematics in the dwarf irregular NGC 1705, Heckman et al. (2001) suggested that the absorption of neutral species could arise in small dense clumps evolving in a superbubble interior. The swept-up clumps have likely the same metallicity as the ionized gas of the $\mathrm{H}$ II region, but more metal-poor gas could lie further away, diluting the integrated abundances. Alternatively, Cannon et al. (2005) proposed that the neutral gas probed by FUV absorption lines comes from a low abundance $\mathrm{H}$ I halo, while metal-rich ionized gas lies close to the stellar clusters. The metallicity offset observed between the neutral gas and the ionized gas seems to be independent of the chemical age of the galaxy, so that abundances have to be kept high in the star-forming regions and low in the halo over extended periods. This implies that star-formation has to remain localized over the galaxy's history.

Our results and calculations (Sect. 6) suggest that most of the metals released by a starburst episode could mix with the $\mathrm{H}$ I region, only increasing slightly its metallicity, while a small fraction (on the order of 1\%) mixes locally within the star-forming regions. It must be stressed that these metals were produced by previous bursts. In such a scenario, low-level star-formation is not required to explain the metallicity of the neutral phase (except if galactic winds allow most of the metals to escape in the intergalactic medium). Is this scenario consistent with the observation of homogeneous abundances in $\mathrm{H}$ II regions of a given star-forming galaxy? The fact that star-forming episodes occur simultaneously indicates that these episodes were probably triggered together, or that they triggered each other over several $10 \mathrm{Myr}$. Hence it is in fact reasonable to expect relatively similar abundances.

The contrasting result of Bowen et al. (2005) in the dwarf spiral galaxy SBS $1543+593$ poses a fundamental question: is the metal deficiency of the H I gas specific to BCDs? Although Lebouteiller et al. (2006) found that the neutral phase toward NGC 604 in the spiral galaxy M 33 is more metal-poor than the ionized gas of the $\mathrm{H}$ II region, i.e., being consistent with results in BCDs, the situation might be different because of the presence of high-velocity clouds along the lines of sight. Rotation in spirals provide an efficient mixing throughout the galaxy. Furthermore, star-formation keeps occurring along the spiral arms. In opposition, BCDs have weak velocity field, and starformation usually occurs in one or several knots (e.g., Papaderos et al. 1996). The different mode of star-formation could be responsible for substantial differences in the dispersal and mixing mechanisms. It seems necessary to study the metal abundance in the neutral gas of dwarf irregular galaxies which are not BCDs. Studies of other star-forming objects thus ought to be performed, which will be possible thanks to the interactive database tool designed by Désert et al. (2006a,b). Analysis is currently in progress. Besides, one of the most complication in the studies of absorption-line spectra of galaxies is the presence of unseen components, possibly saturated. This caveat is usually solved by the use of the weakest observed lines. In that vein, the upcoming installation of the Cosmic Origin Spectrograph onboard the Hubble Space Telescope will allow the sulfur abundance determination in the neutral phase through the interestingly weak S II $\lambda 1256$ multiplet (Pettini \& Lipman 1995). The oxygen abundance will be measured with a better accuracy than with FUSE thanks to the O I $\lambda 1356$ line (Meyer et al. 1998). Finally, the examination of phosphorus lines will confirm its the possible use of phosphorus abundance as a metallicity tracer as suggested by Lebouteiller et al. (2005). This is particularly important for the analysis of the FUSE database since O I lines are generally saturated or close to saturation. Finally, it must be stressed that the ideal case study for deriving abundances in the neutral gas of BCDs is to observe the ISM toward a quasar line of sight (Kunth $\&$ Sargent 1986). The method was successfully used in the dwarf spiral galaxy SBS $1543+593$ by Bowen et al. (2005) but unfortunately, no alignment BCD-quasar has been observed so far. The study of such an alignment would cancel the selection effect in FUSE spectra to probe gas toward star-forming regions within the galaxy.

\section{Summary and conclusions}

We have analyzed the FUV FUSE absorption spectrum of the BCD Pox 36. The following results are found:

- We detect the interstellar absorption lines of H I, N I, O I, Si II, P II, Ar I, and Fe II. Pox 36 appears to be have a particularly low foreground gas content, which allows us to measure column densities with minimal systematic errors due to hidden saturation effects. 


\section{$22000 \mathrm{~K}$}
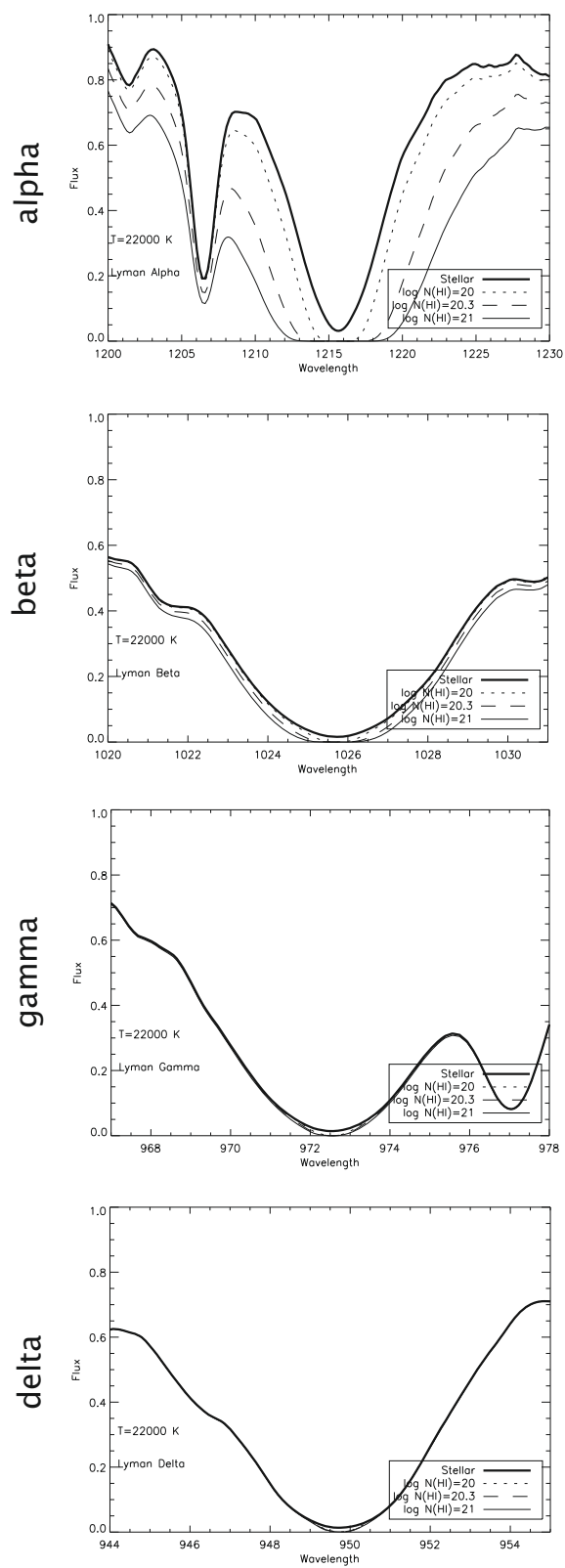

$30000 \mathrm{~K}$
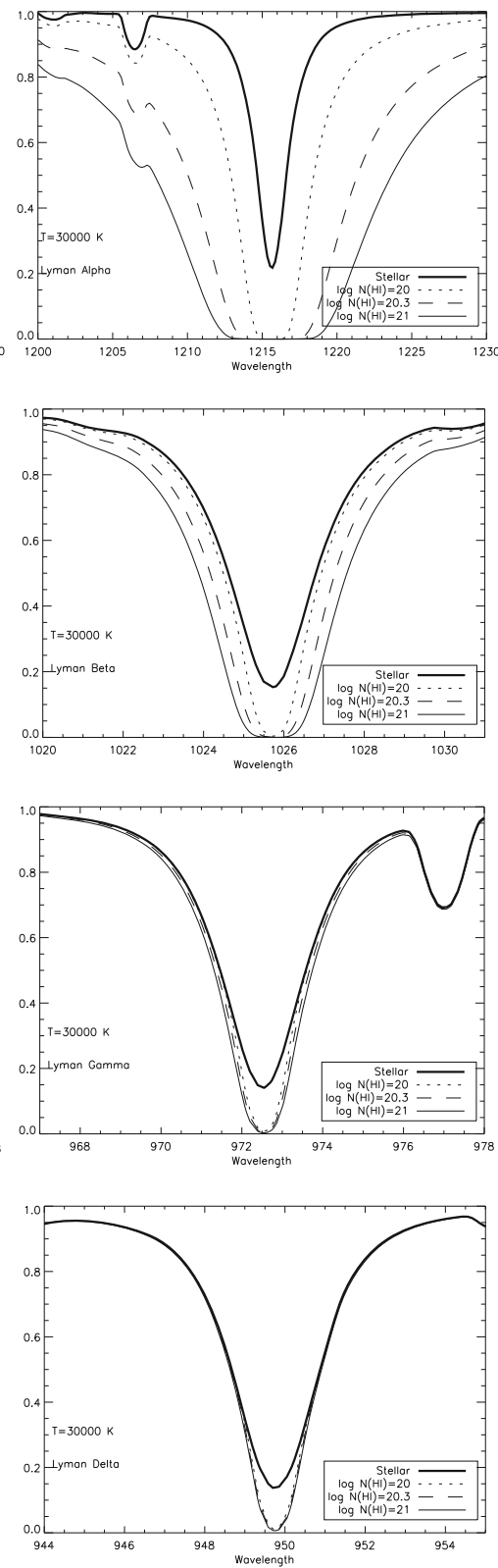

$40000 \mathrm{~K}$
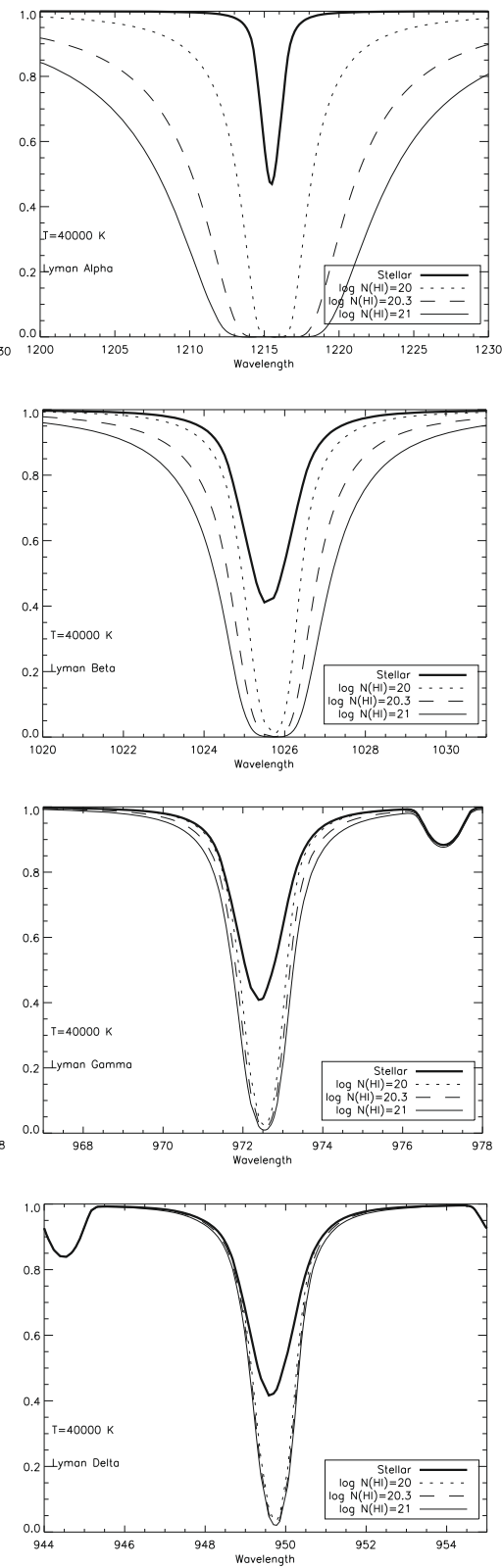

Fig. A.1. Prediction of the total H I profile (stellar and interstellar) of the Lyman lines. All stellar models are for $1 / 1000 Z_{\odot}$ metallicity except for the model at $22000 \mathrm{~K}$ for which we take $1 / 5 Z_{\odot}$.

- Metal column densities have been derived from the line profiles. The interstellar $\mathrm{H}$ I lines are strongly contaminated by stellar absorption. We have modeled the spectral continuum using TLUSTY models. The best model is constrained by the high-order Lyman line Ly $\epsilon$ and gives a photospheric temperature of $\approx 30000 \mathrm{~K}$ (B0 type). After correcting Ly $\beta$ for stellar absorption, we have used the interstellar damping wings to infer a $\mathrm{H}$ I column density equal to $20.3 \pm 0.4$.

- The observed FUV continuum level is consistent with $~ 300$ B0 stars. This represents only the unextincted stars.

- Abundances have been computed from the column densities using the dominant ionization stage. The large $[\mathrm{O} / \mathrm{Fe}]$ ratio in the ionized gas of Pox 36 is interpreted as caused by iron depletion onto dust grains. In contrast, the neutral gas does not show any depletion, which is attributed to the low dust content along the observed lines of sight.
- We find that the neutral gas is metal-underabundant by a factor 5 to 8 as compared to the ionized gas of the $\mathrm{H}$ II regions. This corresponds to a metallicity of $\approx 1 / 35 Z_{\odot}$ in the neutral gas as compared to $\approx 1 / 5 Z_{\odot}$ in the ionized gas and imply that the neutral gas is less chemically processed.

- It is shown that the metal deficiency in the neutral gas can be achieved and maintained if most of the metals released by star-formation episodes mix with the $\mathrm{H}$ I gas, increasing only slightly its metallicity, while a small fraction (1\%) could mix locally and enrich greatly the ionized gas. The metals should come from previous bursts which occurred around the same location as the present one.

- Based on the sample of the seven BCDs studied by FUSE, we have confirmed that there is a systematic underabundance by a factor $\sim 10$ of the neutral gas as compared to the ionized gas in the $\mathrm{H}$ II regions. Besides, $[\mathrm{Ar} / \mathrm{H}]$ and $[\mathrm{N} / \mathrm{H}]$ seem 
$50000 \mathrm{~K}$
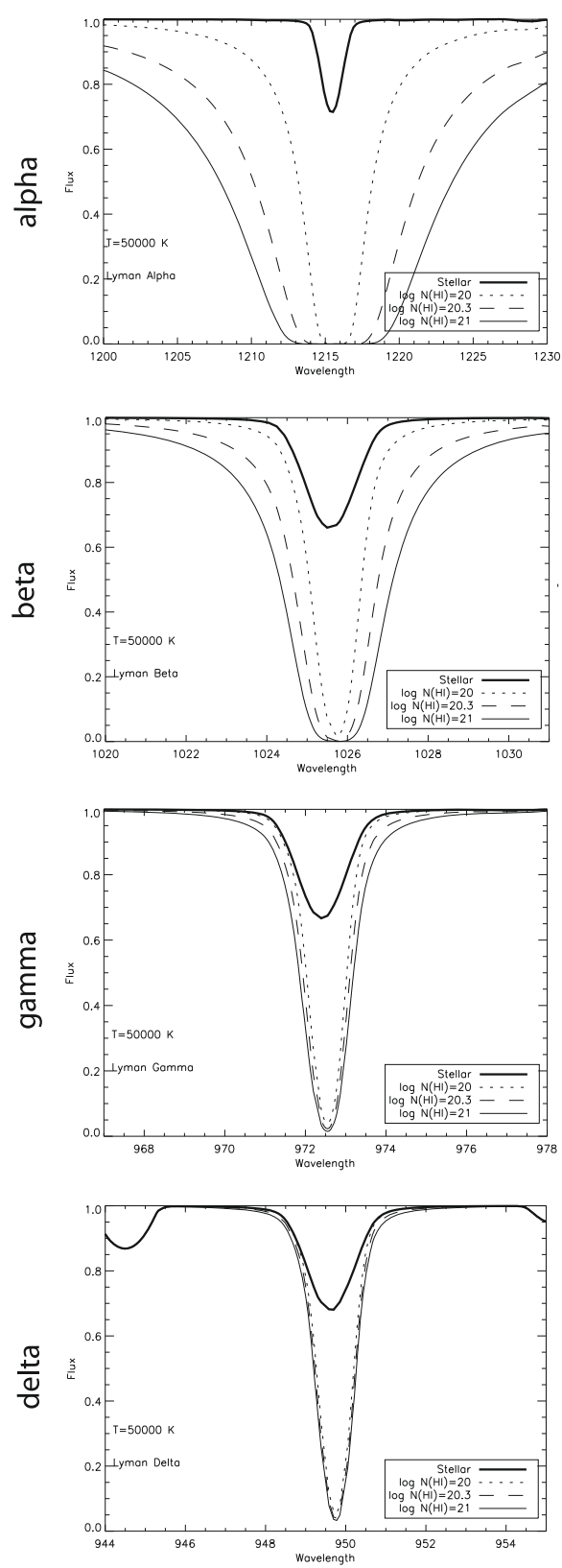

$55000 \mathrm{~K}$
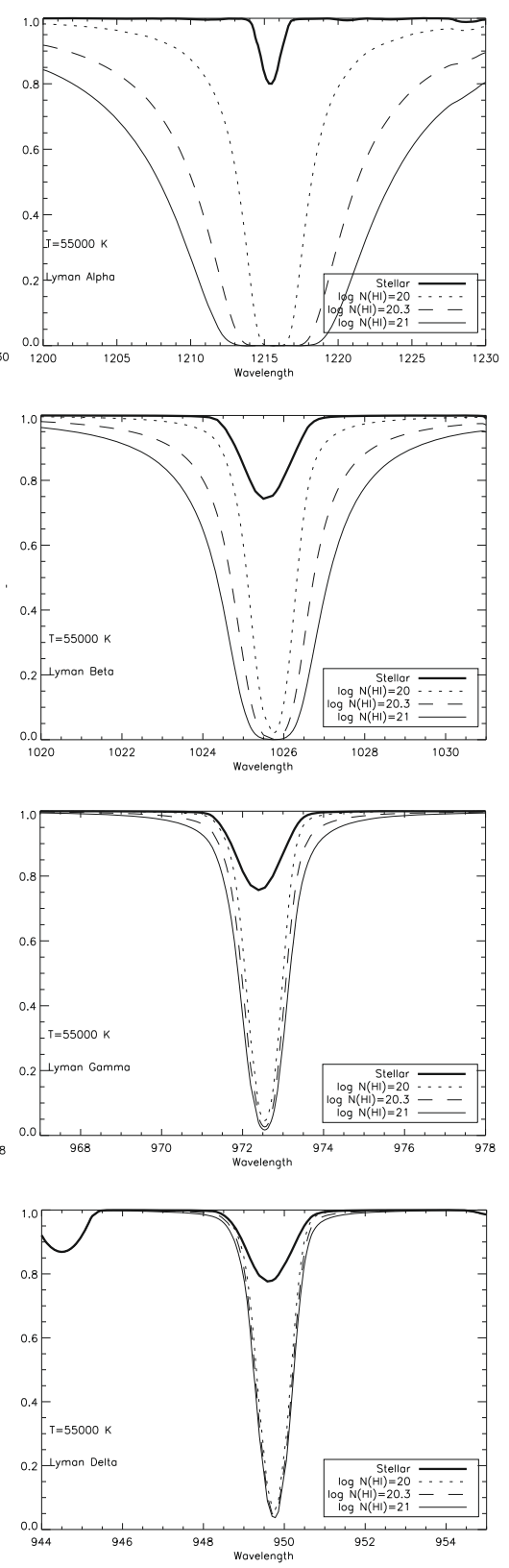

Fig. A.2. See Fig. A.1 for the plot description.

to reach a minimum value for the lowest-metallicity objects $\left(\sim 1 / 8 Z_{\odot}\right)$, which could be due to a default enrichment.

- There is no convincing evidence that the neutral gas in BCDs contains pristine gas pockets, i.e., completely metal-free. However, it could be that the neutral gas of BCDs has not been affected yet by the star-formation episodes, and that its metallicity is that of the IGM.

Acknowledgements. The authors thank A. Lecavelier des Etangs, G. Hébrard, R. Ferlet, and A. Vidal-Madjar for their input as to the handling of the data. T.X.T. acknowledges the support of National Science Foundation grant AST02-05785. He thanks the hospitality of the Institut d'Astrophysique de Paris where part of this work was carried out.

\section{Appendix A: Synthetic H I profiles}

We built $\mathrm{H}$ I profiles of $\operatorname{Ly} \alpha, \beta, \gamma$, and $\delta$ by summing the optical depths of the stellar models and of synthetic interstellar lines.
Profiles were calculated for various column densities and allow one to know, depending on the stellar temperature, which line can be safely used to constrain the interstellar H I column density. From the results of Figs. A.1 and A.2, it is clear that:

- Ly $\alpha$ can be used for any stellar temperature;

- Ly $\beta$ can be used for $T \gtrsim 30000 \mathrm{~K}$;

- Ly $\gamma$ and Ly $\delta$ do not provide any constraint, even for high stellar temperatures. This is because these lines are saturated without any damping wings.

\section{References}

Aloisi, A., Tosi, M., \& Greggio, L. 1999, AJ, 118, 302

Aloisi, A., Savaglio, S., Heckman, T. M., et al. 2003, ApJ, 595, 760

Asplund, M., Grevesse, N., \& Sauval, A. J. 2005, Cosmic Abundances as

Records of Stellar Evolution and Nucleosynthesis, 336, 25

Bowen, D. V., Jenkins, E. B., Pettini, M., \& Tripp, T. M. 2005, ApJ, 635, 880 
Brinchmann, J., Kunth, D., \& Durret, F. 2008, A\&A, 485, 657

Campbell, A. 1988, ApJ, 335, 644

Campbell, A. 1992, ApJ, 401, 157

Cannon, J. M., Skillman, E. D., Sembach, K. R., \& Bomans, D. J. 2005, ApJ, 618,247

Cen, R., \& Ostriker, J. P. 1999, ApJ, 519, L109

Désert, J.-M., Lebouteiller, V., Hébrard, G., et al. 2006a, Astrophysics in the Far Ultraviolet: Five Years of Discovery with FUSE, 348, 547

Désert, M., Hébrard, G., Lecavalier Des Étangs, A., et al. 2006b, Astronomical Data Analysis Software and Systems XV, 351, 475

Doyle, M. T., Drinkwater, M. J., Rohde, D. J., et al. 2005, MNRAS, 361, 34

Dufour, R. J. 1986, PASP, 98, 1025

Field, G. B., Goldsmith, D. W., \& Habing, H. J. 1969, ApJ, 155, L149

Hébrard, G., \& Moos, H. W. 2003, ApJ, 599, 297

Hébrard, G., Lemoine, M., Vidal-Madjar, A., et al. 2002, ApJS, 140, 103

Heckman, T. M., Sembach, K. R., Meurer, G. R., et al. 2001, ApJ, 554, 1021

Hidalgo-Gámez, A. M., Masegosa, J., \& Olofsson, K. 2001, A\&A, 369, 797

Hirashita, H., Tajiri, Y. Y., \& Kamaya, H. 2002, A\&A, 388, 439

Hoopes, C. G., Sembach, K. R., Heckman, T. M., et al. 2004, ApJ, 612, 825

Howk, J. C., Sembach, K. R., Roth, K. C., \& Kruk, J. W. 2000, ApJ, 544, 867

Hummer, D. G., \& Storey, P. J. 1987, MNRAS, 224, 801

Izotov, Y. I., \& Thuan, T. X. 1999, ApJ, 511, 639

Izotov, Y. I., \& Thuan, T. X. 2004, ApJ, 602, 200

Izotov, Y. I., \& Thuan, T. X. 2007, ApJ, 665, 1115

Izotov, Y. I., Thuan, T. X., \& Guseva, N. G. 2005, ApJ, 632, 210

Izotov, Y. I., Stasińska, G., Meynet, G., Guseva, N. G., \& Thuan, T. X. 2006 A\&A, 448, 955

Jenkins, E. B. 1996, ApJ, 471, 292

Kippenhahn, R., \& Weigert, A. 1990, Stellar Structure and Evolution, XVI (Berlin, Heidelberg, New York: Springer-Verlag), A\&A Library, 468, 192

Kobulnicky, H. A., \& Skillman, E. D. 1996, ApJ, 471, 211

Kobulnicky, H. A., \& Skillman, E. D. 1997, ApJ, 489, 636

Kruk, J. W., Howk, J. C., André, M., et al. 2002, ApJS, 140, 19

Kunth, D., \& Sargent, W. L. W. 1983, ApJ, 273, 81

Kunth, D., \& Joubert, M. 1985, A\&A, 142, 411

Kunth, D., \& Sargent, W. L. W. 1986, ApJ, 300, 496

Kunth, D., \& Östlin, G. 2000, A\&ARv, 10, 1

Kunth, D., Sargent, W. L. W., \& Kowal, C. 1981, A\&AS, 44, 229

Kunth, D., Lequeux, J., Sargent, W. L. W., \& Viallefond, F. 1994, A\&A, 282, 709

Lang, K. R. 1992, Astrophysical Data I, Planets and Stars, X (Berlin: Heidelberg, New York: Springer-Verlag), 937, 33

Lanz, T., \& Hubeny, I. 2003, ApJS, 146, 417

Lanz, T., \& Hubeny, I. 2007, ApJS, 169, 83

Lebouteiller, V., Kunth, D., Lequeux, J., et al. 2004, A\&A, 415, 55

Lebouteiller, V., Kuassivi, \& Ferlet, R. 2005, A\&A, 443, 509

Lebouteiller, V., Kunth, D., Lequeux, J., et al. 2006, A\&A, 459, 161

Lecavelier des Etangs, A., Désert, J.-M., Kunth, D., et al. 2004, A\&A, 413, 131

Lee, H., Skillman, E. D., \& Venn, K. A. 2006, ApJ, 642, 813

Legrand, F., Kunth, D., Roy, J.-R., Mas-Hesse, J. M., \& Walsh, J. R. 2000, A\&A, 355,891

Lemoine, M., Vidal-Madjar, A., Hébrard, G., et al. 2002, ApJS, 140, 67

Lequeux, J., Maucherat-Joubert, M., Deharveng, J. M., \& Kunth, D. 1981, A\&A, 103,305

Lisenfeld, U., \& Ferrara, A. 1998, ApJ, 496, 145

Lo, K. Y., Sargent, W. L. W., \& Young, K. 1993, AJ, 106, 507

López-Sánchez, Á. R., Esteban, C., García-Rojas, J., Peimbert, M., \& Rodríguez, M. 2007, ApJ, 656, 168
Martin, C. L., Kobulnicky, H. A., \& Heckman, T. M. 2002, ApJ, 574, 663 Mas-Hesse, J. M., Kunth, D., Tenorio-Tagle, G., et al. 2003, ApJ, 598, 858 McKee, C. F., \& Ostriker, J. P. 1977, ApJ, 218, 148

Meyer, D. M., Jura, M., \& Cardelli, J. A. 1998, ApJ, 493, 222

Meyer, M. J., Zwaan, M. A., Webster, R. L., et al. 2004, MNRAS, 350, 1195

Meynet, G., \& Maeder, A. 2002, A\&A, 390, 561

Moos, H. W., Cash, W. C., Cowie, L. L., et al. 2000, ApJ, 538, L1

Morton, D. C. 2003, ApJS, 149, 205

Noeske, K. G., Guseva, N. G., Fricke, K. J., et al. 2000, A\&A, 361, 33

Östlin, G. 2000, ApJ, 535, L99

Papaderos, P., Loose, H.-H., Thuan, T. X., \& Fricke, K. J. 1996, A\&AS, 120, 207

Pettini, M., \& Lipman, K. 1995, A\&A, 297, L63

Recchi, S., Matteucci, F., \& D'Ercole, A. 2001, MNRAS, 322, 800

Recchi, S., Matteucci, F., D’Ercole, A., \& Tosi, M. 2004, A\&A, 426, 37

Rieschick, A., \& Hensler, G. 2001, A\&ASS, 277, 111

Rieschick, A., \& Hensler, G. 2003, Ap\&SS, 284, 861

Robert, C., Pellerin, A., Aloisi, A., et al. 2003, ApJS, 144, 21

Rodgers, A. W., Peterson, B. A., \& Harding, P. 1978, ApJ, 225, 768

Rodríguez, M., \& Esteban, C. 2004, Recycling Intergalactic and Interstellar Matter, 217, 198

Russell, S. C., \& Dopita, M. A. 1990, ApJS, 74, 93

Sahnow, D. J., Moos, H. W., Ake, T. B., et al. 2000, ApJ, 538, L7

Sargent, W. L. W., \& Searle, L. 1970, ApJ, 162, L155

Savage, B. D., \& Sembach, K. R. 1996, ARA\&A, 34, 279

Schlegel, D. J., Finkbeiner, D. P., \& Davis, M. 1998, ApJ, 500, 525

Schwartz, C. M., \& Martin, C. L. 2004, ApJ, 610, 201

Skillman, E. D., \& Kennicutt, R. C., Jr. 1993, ApJ, 411, 655

Sofia, U. J., \& Jenkins, E. B. 1998, ApJ, 499, 951

Sofia, U. J., Cardelli, J. A., \& Savage, B. D. 1994, ApJ, 430, 650

Telfer, R. C., Kriss, G. A., Zheng, W., Davidsen, A. F., \& Tytler, D. 2002, ApJ, 579,500

Tenorio-Tagle, G. 1996, AJ, 111, 1641

Theureau, G., Bottinelli, L., Coudreau-Durand, N., et al. 1998, A\&AS, 130, 333

Thuan, T. X., \& Martin, G. E. 1981, ApJ, 247, 823

Thuan, T. X., \& Izotov, Y. I. 2005, ApJS, 161, 240

Thuan, T. X., Izotov, Y. I., \& Lipovetsky, V. A. 1997, ApJ, 477, 661

Thuan, T. X., Lecavelier des Etangs, A., \& Izotov, Y. I. 2002, ApJ, 565, 941

Thuan, T. X., Bauer, F. E., Papaderos, P., \& Izotov, Y. I. 2004, ApJ, 606, 213

Thuan, T. X., Lecavelier des Etangs, A., \& Izotov, Y. I. 2005, ApJ, 621, 269

Valls-Gabaud, D. 1993, ApJ, 419, 7

Vanzi, L., \& Sauvage, M. 2006, A\&A, 448, 471

Vanzi, L., Hunt, L. K., Thuan, T. X., \& Izotov, Y. I. 2000, A\&A, 363, 493

Venn, K. A., Lennon, D. J., Kaufer, A., et al. 2001, ApJ, 547, 765

Verhamme, A., Schaerer, D., \& Maselli, A. 2006, A\&A, 460, 397

Vidal-Madjar, A., Kunth, D., Lecavelier des Etangs, A., et al. 2000, ApJ, 538, L77

Vigroux, L., Stasińska, G., \& Comte, G. 1987, A\&A, 172, 15

Wakker, B. P., Savage, B. D., Sembach, K. R., et al. 2003, ApJS, 146, 1

Walsh, J. R., \& Roy, J.-R. 1989, MNRAS, 239, 297

Walsh, J. R., \& Roy, J.-R. 1993, MNRAS, 262, 27

Welch, G. A. 1970, ApJ, 161, 821

Wolfire, M. G., Hollenbach, D., McKee, C. F., Tielens, A. G. G. M., \& Bakes, E. L. O. 1995, ApJ, 443, 152

Zwaan, M. A., Meyer, M. J., Webster, R. L., et al. 2004, MNRAS, 350, 1210 Zwicky, F. 1966, ApJ, 143, 192 